01

\title{
Об излучении гармоник в рентгеновских лазерах на свободных электронах с изменяемым параметром дипольности ондуляторов
}

\author{
(C) К. Жуковский \\ Московский государственный университет им. М.В. Ломоносова, \\ 119991 Москва, Россия \\ e-mail: zhukovsk@physics.msu.ru
}

Поступило в Редакцию 3 марта 2021 г.

В окончательной редакции 8 апреля 2021 г.

Принято к публикации 13 апреля 2021 г.

Рентгеновские лазеры на свободных электронах (ЛСЭ) генерируют короткие импульсы в диапазоне волн 1-100 А. Нами исследована возможность генерации гармоник в рентгеновском диапазоне в ЛСЭ с изменяемым параметром дипольности ондуляторов. Теоретически исследовано излучение гармоник ЛСЭ FLASH 2, ЛСЭ SwissFEL и строящегося ЛСЭ LCLS-II с целью получить максимальную частоту излучения с пучком электронов с минимальной энергией. Проведен сравнительный анализ эффективности использования гармоник в ЛСЭ с самоусилением спонтанного излучения (SASE), в ЛСЭ с усилением затравочного лазерного излучения гармоник банчера (HLSS), и в ЛСЭ с усилением гармоник и подавлением основного тона. Теоретические результаты для излучения каскадного ЛСЭ FLASH2 сравниваются с экспериментальными данными. Показано, что использование выделенного банчера в ЛСЭ с изменяемым параметром дипольности позволяет получить более ранний и быстрый рост мощности гармоник по длине ондуляторов за счет эффективной группировки электронов на длинах волн гармоник. На основание этого вывода в ЛСЭ SwissFEL предложено использовать отличные друг от друга значения параметра дипольности в ондуляторах для банчера и для усилителя третьей гармоники. Теоретически показано, что это позволит сократить длину ЛСЭ и повысить частоту излучения на $\sim 30 \%$. Исследована возможность генерации гармоник в строящемся ЛСЭ LCLS-II в трех возможных режимах: SASE, HLSS и усиления третьей гармоники ЛСЭ с одновременным подавлением основного тона. Показано, что преимущество выделенного банчера в LCLS-II не может быть полностью использовано с имеющимися ондуляторами с изменяемым параметром $k$ из-за невысокой эффективности усиления гармоник в ондуляторах каскадов усилителя с малым значением $k=0.6$. В качестве альтернативного решения предложено использовать третью гармонику в ондуляторах с $k=2.24$ по всей длине ЛСЭ LCLS-II, и подавление основного тона путем нарушения его банчинга между ондуляторами. Продемонстрировано, что такая схема позволяет получить излучение мощностью более $1 \mathrm{GW}$ на длине волны третьей гармоники $\lambda \approx 0.25 \mathrm{~nm}$ уже на $\sim 40$ m ондуляторов с пучком с энергией $E=4 \mathrm{GeV}$.

Ключевые слова: ондуляторное излучение, лазер на свободных электронах, гармоники, рентгеновское излучение.

DOI: $10.21883 / J T F .2021 .12 .51752 .51-21$

\section{Введение}

Лазеры на свободных электронах (ЛСЭ) в XXI веке все чаще используются как инструмент исследования в разных областях науки, а не только как экспериментальные установки. В ЛСЭ взаимодействие ондуляторного излучения (ОИ) с электронами в ондуляторе приводит к группировке электронов на длине волны излучения и его гармоник; теория ЛСЭ была разработана Мадэйем (Madey) [1]. Под его руководством был впервые построен работающий ЛСЭ, который излучал в миллиметровом диапазоне [2]. Сама идея о возможности некогерентного и когерентного излучений электронов в пространственно периодическом магнитном поле была высказана ранее Гинзбургом [3] в 1947 г;; вскоре после этого, некогерентное ОИ было получено Мотцом (Motz) [4,5]. Продвижение ЛСЭ в область рентгеновского диапазона в последнее десятилетие связано с развитием техники и технологий. Современные рентгеновские ЛСЭ излу- чают сверхкороткие импульсы когерентного излучения длительностью в фемто- и пикосекунды на длине волны одного ангстрема и менее; пиковая мощность ЛСЭ достигает десятков гигаватт [6-9]. Вследствие совокупности причин теоретического и технического характера, размеры этих ЛСЭ достигают $\sim 1 \mathrm{~km}$, а длина самих ондуляторов в ЛСЭ может превышать $100 \mathrm{~m}$. Для излучения в рентгеновском диапазоне требуются высококачественные пучки электронов высоких энергий $E \sim 5-15 \mathrm{GeV}$, что во многом обусловливает большие размеры установок. Кроме того, для эффективной группировки электронов в однопроходных ЛСЭ требуется большая плотность тока. Это возможно при сильном токе в ондуляторах, $I_{0} \sim 1 \mathrm{kA}$, и малом сечении пучка, $\sigma_{x, y} \sim 10-30 \mu \mathrm{m}$, с малой расходимостью. Кроме малого нормализованного эмиттанса пучка, $\gamma \varepsilon_{x, y} \sim 1 \mathrm{~mm} \mathrm{mrad}$, нужен также малый разброс энергий, $\Delta \gamma / \gamma \sim 10^{-4}$. Эти требования удалось выполнить и построить первый рентгеновский ЛСЭ LCLS в США в 2009 г. [10-12]. 
В рентгеновском диапазоне в отсутствие готовых источников когерентного затравочного излучения используют однопроходные ЛСЭ с самоусилением спонтанного излучения (self-amplified spontaneous emission, SASE), которое развивается из случайных начальных когерентных флуктуаций шума электронного банча. Это когерентное излучение усиливается за один проход в длинных ондуляторах. Излучение первых ондуляторных секций может быть отфильтровано на монохроматоре или другим образом, и использовано в качестве затравочного для последующих ондуляторных секций. Кроме того, гармоники излучения первых секций тоже могут использоваться для затравки и усиления в последующих каскадах усиления. Использование гармоник ОИ в принципе позволяет уменьшить требуемую энергию электронов и, как следствие, уменышить размеры и стоимость установки. Излучение в режиме SASE зарождается из случайного процесса - шума, и его временная когерентность несравнимо хуже той, что дает внешний затравочный лазер. Использование каскадного усиления и умножения гармоник позволяет снизить требуемую частоту затравочного излучения и использовать для него готовый, например, эксимерный лазер. Каскадное усиление высших гармоник ОИ в ЛСЭ (high gain harmonic generation, HGHG FEL) с затравочным лазером было предложено в $[13,14]$. Наряду с этим в ЛСЭ также используют эхо нескольких затравочных лазеров, которые последовательно модулируют энергию электронного банча [15]; этот принцип реализован, например, в $[16,17]$. Возможен случай, когда гармоника в ондуляторе не излучается, но группировка электронов на ее длине волны создает эквивалентную мощность для усиления в следующем ондуляторе, настроенном в резонанс $[18,19]$; возможно также использование нескольких каскадов для умножения гармоник [20-22]. Применение монохроматоров или иная фильтрация излучения между каскадами ЛСЭ, настроенных на гармоники друг друга, позволяет получить от первых ондуляторов в режиме SASE затравочное лазерное излучение для последующих каскадов ЛСЭ (harmonic lasing selfseed, HLSS); этот принцип реализован, например, в ЛСЭ FLASH2 с изменяемым параметром дипольности $k$ ондуляторов [23,24]. Ниже мы рассмотрим в числе других излучение этого ЛСЭ и сравним наши теоретические результаты с имеющимися экспериментальными данными. Мы также рассмотрим возможность использования раздельных банчера и усилителя гармоник в новом тестируемом ЛСЭ SwissFEL [25-29] с изменяемым параметром $k$, и в новейшем строящемся ЛСЭ LCLS-II [30-33].

В следующих разделах приводится краткое аналитическое описание ОИ и эволюции мощности гармоник ОИ в однопроходном ЛСЭ. Далее рассматриваются некоторые эксперименты с ЛСЭ и проверяется развитая теория. После этого приводится исследование возможности использования гармоник в ЛСЭ SwissFEL и LCLS-II с целью получить максимальное их усиление, моделируется и сравнивается их излучение в различных режимах.

\section{1. Спонтанное ОИ}

Теория ОИ хорошо изучена и представлена во множестве книг и статей [34-40] и др. Простейшая модель поля ондулятора представляет собой чисто синусоидальное магнитное поле, $\mathbf{H}=H_{0}\left(0, \sin \left(k_{\lambda} z\right), 0\right)$, где и $k_{\lambda}=2 \pi / \lambda_{u}$ и $\lambda_{u}$ - период ондулятора. Такая модель неплохо описывает поле вблизи оси ондулятора, но не удовлетворяет уравнениям Максвелла во всем зазоре ондулятора. В результате поперечных колебаний электрона на фоне его релятивистского дрифта вдоль оси генерируются гармоники с частотами $\omega_{n}=2 \pi c / \lambda_{n}$ и длинами волн

$$
\lambda_{n}[\mathrm{~nm}]=1.3056 \frac{\lambda_{u}[\mathrm{~cm}]}{n E^{2}\left[\mathrm{GeV}^{2}\right]}\left(1+\frac{k^{2}}{2}+(\gamma \theta)^{2}\right),
$$

где $k=H_{0} \lambda_{u} e / 2 \pi m c^{2} \approx 0.9337 \lambda_{u}[\mathrm{~cm}] H_{0}[\mathrm{~T}]-$ ондуляторный параметр, $e$ - заряд электрона, $m$ - масса электрона, $H_{0}$ - напряженность магнитного поля, $c$ скорость света, $E$ - энергия электрона, $\gamma=E / m c^{2}-$ релятивистский фактор, $\theta-$ угол между направлением релятивистского дрифта электрона и направлением на наблюдателя. В плоском ондуляторе с идеальным синусоидальным полем и идеально узким пучком электронов на оси излучаются только нечетные гармоники ОИ; в реальных устройствах на оси присутствуют также четные гармоники. Спектрально-угловое распределение интенсивности излучения $n$-й гармоники ОИ в ондуляторе с $N$ периодами длины $\lambda_{u}$ определяется хорошо известным выражением

$$
\frac{d^{2} I}{d \omega d \Omega}=\frac{e^{2} N^{2} \gamma^{2}}{c} \frac{k^{2} \sum_{n=-\infty}^{\infty} n^{2} S_{N}^{2}\left(\left|f_{n, x}\right|^{2}+\left|f_{n, y}\right|^{2}\right)}{\left(1+\left(k^{2} / 2\right)+(\gamma \theta)^{2}\right)^{2}},
$$

где $S_{N}=\left(\sin v_{n}\right) / v_{n}, v_{n}=\pi N n\left(\left(\lambda_{n} / \lambda\right)-1\right)$ - параметр расстройки относительно резонансов ОИ (1), $f_{n, x, y}-$ коэффициенты Бесселя $x$ - и $y$-поляризаций ОИ. Коэффициенты Бесселя зависят от поля в ондуляторе; аналитические выражения для $f_{n}$ в различных ондуляторах, в том числе с гармониками поля, были рассчитаны и приведены, например, в [41-47]. Разброс энергий электронов в пучке уменьшает интенсивность ОИ, в особенности для высших гармоник ОИ. Аналитически это можно учесть в следующей свертке: $\quad \int_{-\infty}^{+\infty} d^{2} I\left(v_{n}+4 \pi n N \varepsilon\right) e^{-\varepsilon^{2} / 2 \sigma_{e}^{2}} d \varepsilon / d \omega d \Omega \sqrt{2 \pi} \sigma_{e}$. Верность такого учета была проверена численно в [48], в том числе для ондуляторов с гармониками поля. Кроме гармоник поля в ондуляторе в той или иной мере всегда присутствуют непериодические магнитные компоненты; они появляются из-за неидеальности магнитов ондулятора и влияния внешних полей, например, магнитного поля Земли. Эти факторы учитываются при конструировании ЛСЭ: внешние поля экранируются, а ошибки намагничивания тщательно компенсируются, так как даже слабое непериодическое поле напряженностью $\sim 10^{-4} H_{0}$ в длинном ондуляторе $\sim 3-5$ м может 
вызвать расстройку когерентности осцилляций электрона по длине ондулятора. Как следствие, возникают красное смещение резонансов относительно расчетных значений (1), четные гармоники на оси ондулятора и уширение линий спектра. Аналитическое исследование этих явлений проведено, например, в $[49,50]$ и других работах. В реальном ондуляторе магнитное поле, удовлетворяющее уравнениям Максвелла во всем зазоре, имеет сложную форму с гиперболическими составляющими [37-40]. В реальном пучке, электроны находятся на конечном расстоянии от его оси. Вследствие этого возникают бетатронные колебания [37-40] с частотой $\Omega_{\beta}=\sqrt{2} \pi c k / \lambda_{u} \gamma$. Они, в свою очередь, приводят к расщеплению каждой линии излучения $n$-й гармоники ОИ на бетатронные гармоники, отстоящие друг от друга на частоту $\omega_{\beta}$ :

$\omega_{\beta}=2 \sqrt{2} \pi c \gamma k / \lambda_{u}\left(1+\left(k^{2} / 2\right)\right), \omega_{\beta} / \omega_{n} \cong k \sqrt{2} n \gamma \propto 1 / \gamma$.

Кроме того, они вызывают четные гармоники ОИ на оси ондулятора. Для релятивистских пучков $\gamma \gg 1$, и тогда бетатронные гармоники находятся очень близко друг к другу: $\omega_{\beta} /\left.\omega_{n}\right|_{\gamma \gg 1} \ll 1$. С учетом этого для $n$-й гармоники плоского ондулятора с единственной периодической компонентой $H_{0} \sin k_{\lambda} z$ получаются известные коэффициенты Бесселя, которые мы запишем в следующем компактном виде:

$$
f_{n, x}=\sum_{p} \tilde{J}_{p}\left|\left(J_{n+1}^{n}+J_{n-1}^{n}\right)+\frac{2}{k} \gamma \theta \cos \varphi J_{n}^{n}\right|
$$

$f_{n, y}=\sum_{p}\left(\tilde{J}_{p}\left|\frac{2}{k} \gamma \theta \sin \varphi J_{n}^{n}\right|+J_{n}^{n} \frac{\sqrt{2} \pi y_{0}}{\lambda_{u}}\left(\tilde{J}_{p+1}-\tilde{J}_{p-1}\right)\right)$,

где $\theta$ и $\varphi-$ соответственно азимутальный и полярный углы, $y_{0}-$ расстояние в пучке от оси ондулятора, $J_{n}^{m}$ и $\tilde{J}_{p}$ - обобщенные функции Бесселя, заданные в интегральном виде:

$$
\begin{gathered}
J_{n}^{m} \equiv J_{n}^{m}(\xi, \xi)=\int_{-\pi}^{\pi} \frac{d \alpha}{2 \pi} e^{i(n \alpha+\xi m \sin \alpha+\xi m \sin (2 \alpha))}, \\
\xi=\theta \cos \varphi \frac{\lambda_{u} k}{n \lambda_{n} \gamma}, \quad \xi=\frac{\lambda_{u} k^{2}}{8 n \lambda_{n} \gamma^{2}}, \\
\tilde{J}_{p} \equiv J_{n=p}^{m=1}(-\kappa,-\eta), \quad \kappa=\frac{4 \pi \theta y_{0} \gamma^{2}}{\lambda_{u}\left(1+\left(k^{2} / 2\right)\right)}, \\
\eta=\frac{\pi^{2} \gamma y_{0}^{2} k}{\sqrt{2} \lambda_{u}^{2}\left(1+\left(k^{2} / 2\right)\right)} .
\end{gathered}
$$

В коэффициентах Бесселя $f_{n}$, нечетные гармоники $\sim J_{n \pm 1}^{n}$, четные $\sim J_{n}^{n}$. Расщепление линии спектра гармоники $n$ на бетатронные гармоники $p$ описывается суммированием по $p$ функций Бесселя $\tilde{J}_{p}$. Отметим, что в соответствующих выражениях (4) для плоского ондулятора присутствуют четные гармоники излучения за счет бетатронного вклада $\sim y_{0}$ и за счет вклада угловых эффектов $\sim \gamma \theta$. Вклад непериодических компонент поля в генерацию четных гармоник ОИ учитывается аналогично угловому вкладу в (4), где в качестве угла $\gamma \theta$ используется значение угла изгиба $\gamma \theta_{H}$, индуцированного непериодическим полем $H_{d}$ [51-55]:

$$
f_{n, y}^{H}=\sum_{p} \tilde{J}_{p} \gamma \theta_{H} \sqrt{3} / k .
$$

Практическая ширина линии излучения определяется составом гармоник $p$ в выражениях (4) для коэффициентов Бесселя. Бесконечная сумма по $p$ в реальности ограничивается конечным числом слагаемых, которые дают значимый вклад в излучение гармоники $n$ ОИ. Число значимых гармоник $p$ для каждой установки определяется функцией типа Бесселя $\tilde{J}_{p}$. В реальных ондуляторах значимые $p$ могут быть в довольно широком диапазоне, от единицы до десятка и более. Результаты для некоторых ондуляторов представлены ниже в разд. 4-6. Сравнение с имеющимися экспериментальными данными показывает отличное согласие теории и экспериментов.

\section{2. Вынужденное излучение в однопроходных ЛСЭ}

Теория вынужденного ОИ была развита Мадэйем (Madey) [1], который рассмотрел в совокупности излучение от электронов и его взаимодействие с самими электронами в пучке. Мадей показал, что группировка электронов в микробанчи в ондуляторе происходит вследствие взаимодействия электронов с волной электромагнитного поля излучения. Физика ЛСЭ в настоящее время хорошо изучена и описана во многих книгах и работах, например, [6-9,56] и др. Принцип усиления в ЛСЭ основан на том, что за время пока релятивистский электрон проходит половину периода ондулятора, фотоны опережают электроны ровно на половину длины волны излучения. В результате обмена энергии между электронами и фотонами электроны, отстающие от узлов волны, получают от нее энергию, а электроны, опережающие узлы волны, отдают энергию волне и тормозятся. Таким образом, электроны группируются на длинах волн резонансов ОИ в ЛСЭ в микробанчи длиной короче длины волны излучения; это излучение экспоненциально усиливается вдоль ондуляторов до насыщения. В отсутствие эффективных и доступных оптических резонаторов в рентгеновском диапазоне применяются в основном однопроходные ЛСЭ, где когерентное излучение формируется за один проход в ондуляторах. В однопроходных ЛСЭ длина ондуляторов может достигать сотни метров. Для того чтобы создать когерентное излучение за его один проход, требуется большой коэффициент усиления. Для этого в свою очередь нужно, 
чтобы большое число электронов излучало когерентно уже в начале ЛСЭ, что возможно, если в ЛСЭ присутствует высокая концентрация электронов и течет сильный ток. ЛСЭ могут работать в режимах усилителя, самоусиления спонтанного излучения, каскадного усиления гармоник и др. В отсутствие готового источника когерентного затравочного излучения в рентгеновском диапазоне обычно используют самоусиление спонтанного излучения, которое зарождается из случайных когерентных осцилляций излучения электронного банча. Детали теории ЛСЭ, которая основана на совместном решении уравнения колебаний и неоднородного уравнения поля электромагнитной волны, приведены во многих работах, например, в [6-9] и др. Основные закономерности прослеживаются уже в одномерном описании по оси ондуляторов. Обычно в первом приближении считают, что разброс энергии электронов пучка и эмиттанс пренебрежимо малы; тогда, для амплитуды электрического поля электромагнитной волны получается дифференциальное уравнение третьего порядка, которое записывается (см. например, [56]) следующим образом:

$$
\frac{d^{3} E}{d z^{3}}-4 i k_{\lambda} \eta \frac{d^{2} E}{d z^{2}}+\left(k_{p}^{2}-4 k_{\lambda}^{2} \eta^{2}\right) \frac{d E}{d z}-i g_{0}^{3} E(z)=0,
$$

где $0<\eta=\left(\gamma-\gamma_{r}\right) / \gamma_{r} \ll 1, \eta$ описывает отклонение $\gamma$-фактора электрона от резонансного значения $\gamma_{r}$, соответствующего длине волны затравочного излучения: $\quad \lambda_{\text {seed }}=\frac{\lambda_{u}}{2 \gamma_{r}^{2}}\left(1+\frac{k_{\mathrm{eff}}^{2}}{2}\right)$ и энергии $W_{r}=\gamma_{r} m c^{2}$. При $\gamma=\gamma_{r}$ длина волны основного тона ОИ (1) на оси совпадает с затравочной длиной волны $\lambda_{\text {seed }}$. В (7) обозначения параметров таковы: $k_{p}=\sqrt{\frac{4 \gamma^{2} c g_{0}^{3}}{\omega k^{2}}}$ и $g_{0}=\left(\frac{\mu_{0} k^{2} e^{2} k_{\lambda} n_{e}}{4 \gamma^{3} m}\right)^{1 / 3}$, где $n_{e}-$ концентрация электронов, $\mu_{0}=1 / c^{2} \varepsilon_{0}=4 \pi 10^{-7}[\mathrm{Vs} / \mathrm{Am}]$ и $\varepsilon_{0}=\frac{10^{7}}{4 \pi c^{2}}\left[\frac{\mathrm{C}}{\mathrm{Vm}}\right]-$ абсолютная диэлектрическая постоянная. Дифференциальное уравнение третьего порядка (7) имеет аналитическое решение, при $\gamma=\gamma_{r}$ это решение содержит три слагаемых $[8,57,58]$ :

$$
E(z)=A_{1} e^{-i g_{0} z}+A_{2} e^{\frac{i+\sqrt{3}}{2} g_{0} z}+A_{3} e^{\frac{i-\sqrt{3}}{2} g_{0} z},
$$

где $g_{0}-$ коэффициент усиления ЛСЭ. В решении (8) уравнения (7) экспоненциальный рост напряженности поля волны по оси $z$ ондуляторов описывается вторым слагаемым с положительной действительной составляющей экспоненты: $A_{2} e^{\frac{i+\sqrt{3}}{2} g_{0} z}$. Ему соответствует экспоненциальный рост мощности излучения $P(z) \propto e^{\sqrt{3} g_{0} z}$. Можно ввести длину усиления ЛСЭ: $L_{g 0}=1 / \sqrt{3} g_{0}$; тогда для мощности имеем: $P(z) \propto e^{z / L_{g 0}}$. В этом контексте определяют безразмерный параметр Пирса $\rho$, связанный с усилением $g_{0}[6-9]: \rho=\left(\lambda_{u} g_{0}\right) / 4 \pi$. Такой же подход в целом справедлив и в отношении гармоник ОИ. Параметр Пирса гармоники $n$ может быть выражен через параметры ондулятора и плотность тока в ЛСЭ следующим образом [6-9]:

$$
\rho_{n}=J^{1 / 3}\left(\lambda_{u} k_{\mathrm{eff}} f_{n}\right)^{2 / 3} / 2 \gamma(4 \pi i)^{1 / 3},
$$

где $J=I_{0} / \Sigma$ - плотность электронного тока $\left[\mathrm{A} / \mathrm{m}^{2}\right]$, $I_{0}$ - ток $[\mathrm{A}], \Sigma=2 \pi \sqrt{\beta_{x} \varepsilon_{x} \beta_{y} \varepsilon_{y}}-$ сечение пучка, $\varepsilon_{x, y}=\sigma_{x, y} \theta_{x, y}$ - эмиттансы пучка, $\sigma_{x, y}=\sqrt{\varepsilon_{x, y} \beta_{x, y}}-$ его размеры, $\theta_{x, y}$ - расходимости, а $\beta_{x, y}=\varepsilon_{x, y} / \theta_{x, y}^{2}-$ параметры Твисса, $i \cong 1.7045 \cdot 10^{4}$ размерная постоянная тока Альфвена $[\mathrm{A}], \lambda_{u}-$ период ондулятора $[\mathrm{m}]$, и $k$ - параметр дипольности. Параметр Пирса $\rho$ является фундаментальным параметром однопроходного ЛСЭ. Он определяет максимальную мощность излучения: $P_{F} \approx \sqrt{2} \rho I_{0} E$, т.е. по существу является показателем эффективности ЛСЭ в терминах отношения мощности излучения $P_{F}$ к мощности электронного пучка $P_{\text {beam }}$ : $\rho \sim P_{F} / P_{\text {beam }}$. Параметр Пирса $\rho$ определяет также длину насыщения ЛСЭ: $L_{s} \sim \lambda_{u} / \rho$. В ЛСЭ с самоусилением спонтанного излучения SASE параметр Пирса дает оценку спектральной плотности излучения вблизи насыщения: $\Delta \omega / \omega \approx \rho$ (см., например, [6-9]).

В ЛСЭ SASE в отсутствие затравочного излучения начальная мощность задается когерентным шумом банча. Средняя мощность случайного когерентного излучения банча в ЛСЭ на длине волна $\lambda$ (см., например, [8]) также определяется параметром Пирса и числом электронов $N_{l_{c}} \approx I \lambda / e c \rho$ в пространственном когерентном интервале $l_{c} \approx \lambda / \rho: P_{\text {noise }} \approx \rho \gamma m c^{2} / N_{l_{c}}$. Характерное значение числа электронов в одном пространственном когерентном интервале $l_{c}: N_{l_{c}} \sim 10^{5}-10^{7}$; соответствующий $l_{c}$ временной когерентный интервал: $\tau_{c} \approx l_{c} / c=\lambda / c \rho$. Длительность электронного банча определяется зарядом банча $Q$ и током: $\tau_{e} \approx Q / I_{0}$. Характерные значение заряда банча и тока в рентгеновских ЛСЭ находятся в широком интервале: $Q \sim 0.01-1 \mathrm{nC}, I_{0} \sim 0.1-10 \mathrm{kA}$; характерная длительность банча обычно составляет $\tau_{e} \sim 10-300 \mathrm{fs}$ и обычно в одном банче содержится несколько когерентных интервалов. Конкретные значения для разных ЛСЭ сильно отличаются друг от друга; так, например, импульс излучения UV-A ЛСЭ LEUTL [59] содержал всего один когерентный интервал $\tau_{\gamma} \approx \tau_{c}$, в других ЛСЭ оценка $\tau_{\gamma} \approx \sqrt{2 \pi} \tau_{e} \sqrt{L_{g} / L_{s}}$ обычно дает 10-50 когерентных интервалов в одном импульсе излучения.

В реальном ЛСЭ нужно учитывать потери, связанные с дифракцией, разбросом энергии и эмиттансом пучка, которые ухудшают группировку электронов, уменьшают усиление ЛСЭ и его мощность и увеличивают длину усиления и насыщения. Аналитический учет потерь был предложен Мингом Кси (Ming Xie) [60,61] в виде корректирующей поправки $\Lambda$, например, для длины усиления: $L_{g}=L_{g 0}(1+\Lambda)$. Это на первый взгляд простое описание, на самом деле сложное, так как $\Lambda$ содержит девятнадцать численных коэффициентов и нецелых степеней семи слагаемых $[60,61]$. Приближенное описание с меньшим числом параметров было предложено в рабо- 
тах Даттоли (G. Dattoli) и соавторов $[18,49,50,62-65]$ и др. Так, с учетом дифракции реальное значение параметра Пирса $\tilde{\rho}$ оказывается несколько меньше, чем $\rho[18]$ :

$$
\tilde{\rho_{n}} \approx \rho_{n} / \sqrt[3]{1+\frac{\lambda_{u} \lambda_{n}}{16 \pi \rho_{n} \Sigma}}
$$

В результате уменьшается максимально возможная мощность $n$-й гармоники в режиме независимого роста ее мощности: $P_{F n} \approx \sqrt{2} \tilde{\rho}_{n} I_{0} E, P_{F} \equiv P_{F, 1}$ и увеличивается длина усиления ЛСЭ: $L_{g} \cong \lambda_{u} \Phi_{1} /(4 \pi \sqrt{3} \tilde{\rho})$ [62-65]. Для гармоник излучения в режиме их нелинейной генерации, индуцированной основным тоном, длина усиления гармоники $L_{n, g}$ и ее мощность насыщения $P_{n, F}$ тем сильнее зависят от потерь, чем больше номер гармоники $n$. Соответствующие приблизительные формулы, учитывающие влияние разброса энергий и эмиттанса пучка на длину усиления и мощности насыщения гармоник, индуцированных основным тоном, были предложены в [62-65]:

$$
L_{n, g} \cong \frac{\lambda_{u}}{4 \pi \sqrt{3} n^{1 / 3} \tilde{\rho}_{n}} \Phi_{n}, \quad P_{n, F}=\eta_{n} \frac{P_{1, F}}{\sqrt{n}}\left(\frac{f_{n}}{n f_{1}}\right)^{2},
$$

где $P_{1, F} \cong \sqrt{2} \tilde{\rho}_{1} I_{0} E \eta_{1} \tilde{\rho}_{1} / \rho_{1}-$ мощность насыщения основного тона и

$$
\begin{gathered}
\mu_{\varepsilon, n} \cong \frac{2 \sigma_{\varepsilon}}{n^{1 / 3} \tilde{\rho_{n}}}, \quad \Phi_{n} \cong\left(\xi^{n}+0.165 \mu_{\varepsilon, n}^{2}\right) e^{0.034 \mu_{\varepsilon, n}^{2}}, \\
\eta_{n} \cong 0.942\left(e^{-\Phi_{n}\left(\Phi_{n}-0.9\right)}+1.57\left(\Phi_{n}-0.9\right) / \Phi_{n}^{3}\right),
\end{gathered}
$$

а параметр $\xi$ зависит от эмиттанса и параметров Твисса $\beta$ сложным образом [62]. Для согласованного качественного пучка влияние $\xi$ мало; в рентгеновских ЛСЭ $\xi \approx 1-1.05$. Длина насыщения основного тона [62]: $L_{s} \cong 1.07 L_{1, g} \ln \left(9 \eta_{1} P_{1, F} / P_{1,0}\right)$, где $P_{1,0}-$ начальная мощность затравочного излучения или шума банча: $P_{\text {noise }} \approx 1.6 \rho_{n}^{2} e 4 \pi c P_{e} /\left(I_{0} \lambda_{n}\right)[66] ;$ коэффициент $1 / 9$ для $P_{1,0}$ отвечает тому, что в начале имеется не сгруппированный пучок. Для эффективной группировки и усиления в ЛСЭ важно высокое качество электронного пучка, который должен иметь малый разброс энергий $\sigma_{e}$, по крайней мере $\Delta \gamma / \gamma<\rho$, обычно $\sigma_{e}<\rho / 2$, и малый эмиттанс $\varepsilon_{x, y} \leq \lambda / 4 \pi$; кроме того, электронный пучок должен быть несколько шире фотонного пучка: $\sigma_{x} \geq \sigma_{\gamma}$. В современных установках удается поддерживать разброс энергий пучка $\sigma_{e}<10^{-3}$ и нормированный эмиттанс $\gamma \varepsilon<1 \mu \mathrm{m}$. Таким образом, параметр Пирса должен быть $10^{-4}<\rho \sim 10^{-3}$; это позволяет получить длину усиления $L_{g} \sim 1-3 \mathrm{~m}$ и разумную длину насыщения $<100 \mathrm{~m}$.

Анализ возможных решений, удовлетворяющих уравнению (8) и описывающих экспоненциальный рост мощности гармоник по длине ондуляторов, был проделан многими авторами. В частности, следующая функция была предложена в [62-64]:

$$
A_{n}(z) \cong \frac{1}{9}\left(3+2 \cosh \frac{z}{L_{n, g}}+4 \cos \frac{\sqrt{3} z}{2 L_{n, g}} \cosh \frac{z}{2 L_{n, g}}\right) .
$$

Функция (13) описывает также запаздывание роста мощности на примерно 1-2 длины усиления в начале ЛСЭ, так как там развиваются процессы модуляции энергии электронов, что приводит к модуляции электронной плотности и группировке электронов на длине волны излучения. За этим следует экспоненциальный рост мощности до насыщения $\bar{P}_{n, F}$; в режиме насыщения происходит последовательная передача энергии от электронов к волне и наоборот, что приводит к осцилляциям мощности с периодом $\sim L_{g} / n$ :

$$
\bar{P}_{n, F} \cong P_{n, F}\left(1+0.3 \cos \left(\frac{n\left(z-L_{s}\right)}{1.3 L_{g}}\right)\right) / 1.3
$$

около среднего значения $\approx P_{n, F}$, определенного в (11). Для описания экспоненциального роста мощности излучения по длине ЛСЭ от начального значения $P_{0}$ до мощности насыщения $P_{F}$ была предложена логистическая функция [65]:

$$
P(z) \cong P_{0} e^{z / L_{s}} /\left(1+\frac{P_{0}}{P_{F}} e^{z / L_{s}}\right) .
$$

В изначально не сгруппированном пучке электронов эффективно используется только $1 / 9$ всей доступной мощности: $P_{0} / 9$, которой можно поставить в соответствие начальное значение банчинга $b_{0}^{2} \cong P_{0} / 9 \rho P_{\text {beam }}$ (см. [6-9] и др.). Описание роста мощности с учетом задержки в начале ЛСЭ из-за развития процессов модуляции энергии и перехода их в модуляцию электронной плотности банча, получается, если вместо экспоненты $e^{z / L_{s}}$ в (15) использовать решение (13) [63-64]:

$$
P_{L, n}(z) \cong P_{0, n} A_{n}(z) e^{0.223 z / L_{s}} /\left(1+\left(A_{n}(z)-1\right) \frac{P_{0, n}}{\bar{P}_{n, F}}\right) .
$$

Приведенные выше формулы описывают рост мощности гармоник независимо друг от друга. Помимо этого развивается нелинейно индуцированный основным тоном вклад высших гармоник $\propto e^{n z / L_{g}}[6,9,18,19]$ и др. Моделирование множества экспериментов ЛСЭ в диапазоне от видимого до рентгеновского привело к следующей феноменологической формуле для описания индуцированного роста мощности гармоник $Q_{n}(z)$ [67]:

$$
Q_{n}(z) \cong \frac{\tilde{P}_{n, 0} e^{n z / L_{g}}}{1+\left(e^{n \frac{z}{L_{g}}}-1\right) \frac{\tilde{P}_{n, 0}}{\tilde{P}_{n, F}}}+\frac{P_{n, 0} e^{n z / L_{g}}}{1+\left(e^{n \frac{z}{L_{g}}}-1\right) \frac{P_{n, 0}}{\widehat{P}_{n, F}}},
$$

где возможны две ступени насыщения с мощностями

$$
\begin{gathered}
\tilde{P}_{n, F}=\left.P_{n, F}\right|_{\substack{n \rightarrow \tilde{n}_{n} \\
\tilde{\Phi}_{n} \rightarrow \tilde{\Phi}_{n} \\
\mu_{\varepsilon, n} \rightarrow \mu_{\varepsilon, n}}}, \quad \tilde{\mu}_{\varepsilon, n} \cong \frac{2 n^{2 / 3} \sigma_{\varepsilon}}{\tilde{\rho}_{n}}, \\
\widehat{P}_{n, F} \cong \frac{P_{n, F}}{P_{n, 0}}\left(0.77+0.23 \cos \frac{n\left(z-L_{s}\right)}{1.3 L_{g}}\right)-\frac{\tilde{P}_{n, F}}{2.5} .
\end{gathered}
$$

Отметим, что в начале ондулятора не обязательно должна присутствовать мощность излучения. Усиление 
может развиваться от эффективной мощности, заданной банчингом $b_{n}$ на длине волны $n$-й гармоники даже в отсутствие излучения на ее длине волны, например, для второй гармоники ОИ плоского ондулятора. Созданная банчингом эффективная мощность гармоники усиливается в следующем ондуляторе, настроенном в резонанс с гармоникой предыдущего ондулятора. Учитывая, что в реальности банчинг гармоник в конце ЛСЭ не доходит до идеального значения единицы, используют феноменологические коэффициенты $c_{n} \approx\{1,1.3,2,5,10\}$ и $d_{n} \approx\{1,3,8,40,120\}$ для приведения модели в соответствие экспериментально наблюдаемым значениям в среднем в большом количестве экспериментов. Начальные мощности гармоник выражаются в терминах начального банчинга $b_{n}^{2} \cong\left(P_{0,1} / 9 P_{e} \tilde{\rho}_{1}\right)^{n}$ таким образом: $P_{n, 0} \cong c_{n} b_{n}^{2} P_{n, F}$ и $\tilde{P}_{n, 0} \cong d_{n} b_{n}^{2} \tilde{P}_{n, F}$, где коэффициенты $c_{n}$ и $d_{n}$, подобранные так, чтобы эволюция банчинга гармоник соответствовала эволюции их мощности и отражала средние экспериментальные значения. В частности, большее значение коэффициентов для высших гармоник описывает их опережающий по сравнению с основным тоном рост и выход на насыщение. Без коррекции, т.е. при $c_{n}=d_{n}=1$, индуцированный рост мощности запаздывает по сравнению с наблюдаемым в экспериментах и численных симуляциях.

В отсутствие начальной мощности излучения, когда есть только сгруппированный пучок электронов с банчингом $b_{0}$, вначале идет квадратичный рост мощности: $P_{c}(z)=\frac{1}{3} \rho b_{0}^{2} P_{\text {beam }}\left(z / L_{g}\right)^{2}[66] ;$ когда мощность излучения достигает значения $P_{0} \approx b_{0}^{2} \rho P_{\text {beam }}$, он сменяется экспоненциальным ростом $\propto P_{0} e^{z / L_{g} 0}$, который соответствует решению уравнения (8). Различные аналитические формулы были предложены для приблизительного описания процесса роста мощности в этом случае (см. $[62,64,66]$ и др.). Например, следующая функция [63]

$$
S_{n}(z) \cong\left|2\left(\cosh \frac{z}{L_{n, g}}-\cos \frac{z}{2 L_{n, g}} \cosh \frac{z}{2 L_{n, g}}\right)\right|,
$$

описывает экспоненциальный рост мощности с небольшой задержкой в начале ондулятора. Используя (19) вместо экспоненты $e^{z / L_{g} 0}$ в (15), получаем хорошее соответствие экспериментам ЛСЭ в режиме экспоненциального роста мощности. Приближенное описание мощности излучения в ондуляторе, куда поступает сгруппированный пучок, дается следующей формулой, которая описывает также осцилляции в режиме насыщения:

$$
P_{L, n}(z) \cong \frac{P_{0, n} S_{n}(z)}{1+\frac{1.3 P_{0, n} S_{n}(z)}{P_{n, F}\left(1+0.3 \cos \left(\left(z-L_{s}\right) / 1.3 L_{n, g}\right)\right)}} .
$$

В ЛСЭ с самоусилением спонтанного излучения SASE, часто используемом в рентгеновском диапазоне, процессы генерации и усиления зарождаются из шума электронного банча, мощность которого оценивается следующим образом: $P_{n \text {,noise }} \approx 6 \pi \rho_{n}^{2} \gamma m_{0} c^{3} / \lambda_{n}$ [66]. В начале такого ЛСЭ присутствует сильная составляющая некогерентного ОИ, так что кривая роста мощности оказывается немного выше экспоненты $P_{0} e^{z / L_{g 0}}$. Это феноменологически описывается следующей добавкой:

$$
N_{n}(z) \approx P_{\text {noise }} \frac{S_{n}(z)}{1+\left(300 S_{n}(z) P_{\text {noise }} / P_{n f}\right)},
$$

которая заметна на первых нескольких длинах усиления и удовлетворительно согласуется с экспериментами на различных ЛСЭ, например, LEUTL [59], SPARC [68], LCLS $[10,12]$ и др. Калибровка формулы (21) проведена по результатам сравнительного анализа с экспериментами в [42,47,53-55]. Добавка мощности в начале ЛСЭ (21) находится в пределах разброса результатов различных численных программ для ЛСЭ; имеется согласие с численными симуляциями, проведенными в [46,69]. Результаты численных программ и измерений мощности гармоник в первых секциях ондуляторов ЛСЭ могут отличаться до 10-100 раз (см. [68] и др.). При этом точность феноменологического описания не уступает численным программам, но аналитическое описание существенно проще численного моделирования, оно не требует специальных компьютерных программ, вычислительных мощностей и квалифицированного персонала для работы с ними. Расчет может быть проделан на любом персональном компьютере с программой аналитических вычислений типа Mathematica или даже на инженерном калькуляторе.

Ниже мы применим представленное выше аналитическое описание к некоторым рентгеновским ЛСЭ с изменяемым параметром дипольности ондуляторов и оценим возможность расширения спектрального диапазона этих ЛСЭ путем использования гармоник.

\section{3. Излучение гармоник в каскадном лСЭ FLASH 2}

Рассмотрим излучение гармоник в ЛСЭ FLASH $2[23,24]$ в диапазоне волн $\lambda \in 4-90 \mathrm{~nm}$. B FLASH 2 используются электроны с энергией $E=0.45-1.2 \mathrm{GeV}$ с разбросом $0.5 \mathrm{MeV}$, заряд банча $Q=0.02-1 \mathrm{nC}$, ток $I_{0}<2.5 \mathrm{kA}$, эмиттанс [23] $\varepsilon_{x, y}=1.4 \mu \mathrm{m} \mathrm{rad} ; \quad$ период ондулятора $\lambda_{u}=3.14 \mathrm{~cm}$; изменяемый зазор ондулятора позволяет варьировать параметр дипольности $k \in[0.707-2.828]$. Ha FLASH 2, пожалуй, впервые реализован каскадный ЛСЭ в рентгеновском диапазоне с затравочным лазерным излучением гармоник первых каскадов HLSS. Кроме того, ЛСЭ FLASH 2 также может генерировать в режиме SASE. Это позволило проанализировать генерацию гармоник в обоих режимах на одной установке и сравнить с теоретическими результатами по формулам разд. 2. В режиме SASE все ондуляторы настроены одинаково; в режиме HLSS последние каскады ондуляторов настроены на вторую или третью гармонику ОИ первых каскадов [23,24]. Например, в эксперименте [23] излучалась длина волны $\lambda=11 \mathrm{~nm}$ от 


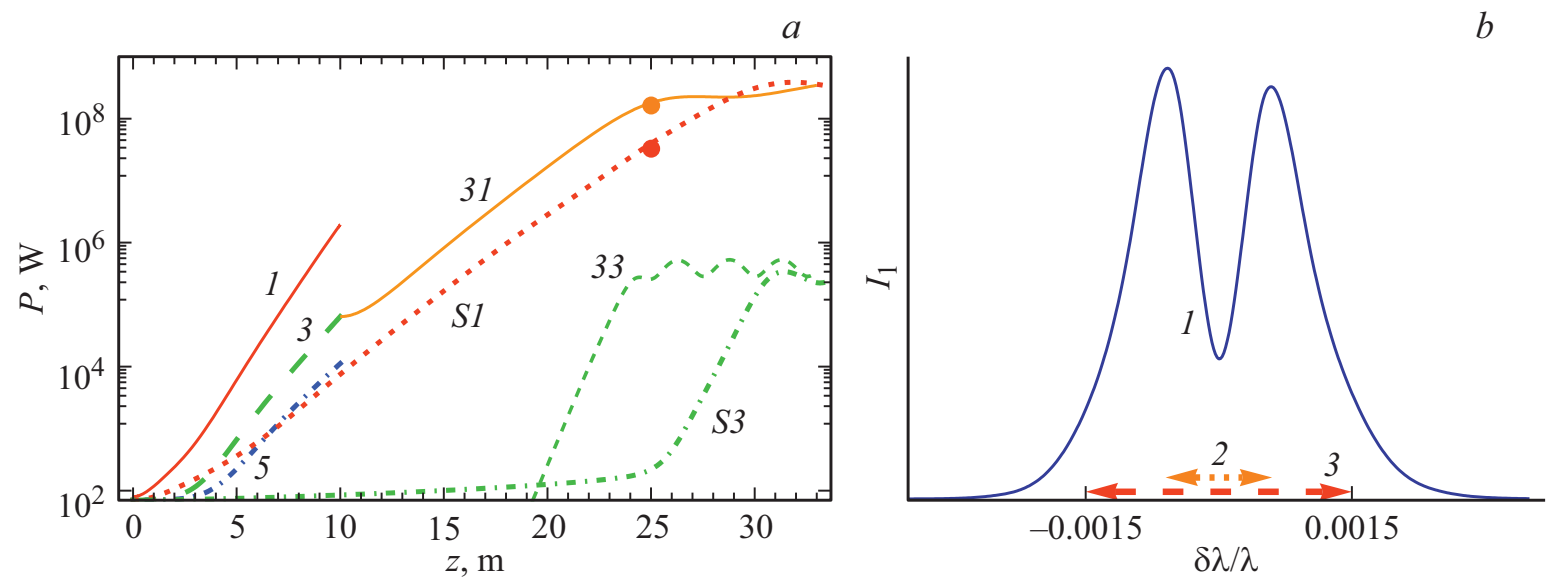

Рис. 1. $a$ - эволюция мощности гармоник в ЛСЭ FLASH 2 с $E=757 \mathrm{MeV}, \sigma_{e}=0.5 \mathrm{MeV}, I_{0}=600 \mathrm{~A}, Q=0.25 \mathrm{nC}$. Гармоники банчера: $1-\lambda_{1}=33 \mathrm{~nm}, 3-\lambda_{3}=11 \mathrm{~nm}, 5-\lambda_{n=5}=6.6 \mathrm{~nm}$; гармоники усилителя: $31-\lambda_{n=1 \times 3}=11 \mathrm{~nm}, 33-\lambda_{n=3 \times 3}=3.7 \mathrm{~nm}$; гармоники SASE: $S 1-\lambda_{\mathrm{SASE}, n=1}=11 \mathrm{~nm}, S 3-\lambda_{\mathrm{SASE}, n=3}=3.7 \mathrm{~nm}$, оранжевая точка (в оnline версии) - мощность, соответствующая измеренной на $25 \mathrm{~m}$ энергии импульса излучения ЛСЭ HLSS $E_{\gamma}=53 \mu \mathrm{J}$, красная точка (в оnline версии) - то же для ЛСЭ SASE $E_{\gamma}=11 \mu \mathrm{J} ; b-$ спектральная плотность излучения $\lambda=11 \mathrm{~nm}$ ЛСЭ FLASH 2: 1 - теоретическая линия спектра, 2 - средняя измеренная спектральная ширина линии, 3 - оценка ширины линии SASE.

электронов с энергией $E=757 \mathrm{MeV}$ в ондуляторах со значением параметра $k=2.687$ в банчере и $k=1.032$ в усилителе третьей гармоники банчера. Расчет показывает, что после $10 \mathrm{~m}$ ондуляторов разброс энергии с учетом индуцированного ЛСЭ вклада незначительно вырос до $\sigma_{e}\left(z_{\text {cut }}=10 \mathrm{~m}\right) \approx 0.07 \% \quad$ и $\quad$ составил половину параметра Пирса: $\rho_{n=3}^{\text {buncher }} \approx \rho_{n=1}^{\text {amp }} \approx 0.0013$. Аналитически рассчитанный нами рост мощности гармоник в этом ЛСЭ показан на рис. 1, $a$. Банч с зарядом $Q=0.25 \mathrm{nC}$ генерировал в режиме каскадного усиления третьей гармоники, фотонный импульс с энергией $E_{\gamma \mathrm{HGHG}}=53 \mu \mathrm{J}$ на $25 \mathrm{~m}$ длины ондуляторов [24]. Мощность импульса $P_{\max }=E_{\gamma} / \tau_{\gamma}$, соответствующая измеренной энергии, показана оранжевой точкой (в online версии) на рис. 1,a. $\mathrm{B}$ режиме SASE все ондуляторы имели значение $k=1.032$ и были настроены на длину волны $\lambda_{1}=11 \mathrm{~nm}$; энергия фотонного импульса на $25 \mathrm{~m}$ длины ондуляторов составила $E_{\gamma}$ SASE $=11 \mu \mathrm{J} \quad[23]$; соответствующая ей мощность отмечена красной точкой (в online версии) на рис. $1, a$. Теоретические результаты соответствуют измерениям, как видно из графиков на рис. 1,a. B режиме HLSS мощность излучения была больше чем в режиме SASE на одинаковой длине ЛСЭ. Это объясняется разными значениями параметров ондуляторов в этих режимах. Так, в ЛСЭ HLSS в ондуляторах банчера с $k \approx 2.7$ достигалась большее усиление третьей гармоники, чем в ондуляторах с $k \approx 1$ в ЛСЭ SASE на той же длине волны. Это видно из сравнения зеленой штриховой линии 1 с пунктирной красной линией $S 1$ (в online версии) на первых 10 m ондуляторов на рис. 1, a. Кроме того, ЛСЭ HLSS оказывается короче ЛСЭ SASE: выход на плато оранжевой сплошной линии 31 на рис. 1, $a$ происходит раньше, чем для красной пунктирной линии $S 1$ (в online версии). Можно сделать вывод, что в ондуляторах ЛСЭ
FLASH 2 с изменяемым параметром $k$, режим усиления высших гармоник HLSS предпочтителен самоусилению спонтанного излучения SASE на той же длине волны.

Ширина линии спектра FLASH 2 рассчитана нами аналитически и показана синей сплошной линией 1 (в online версии) на рис. $1, b$; рассчитанная спектральная плотность, $\Delta \lambda / \lambda \sim 3 \cdot 10^{-3}$, соответствует заявленным в [24] значениям в диапазоне $0.3-0.4 \%$ и показана красной штриховой линией 2 (в online версии) на рис. $1, b$. Это подтверждает правильность нашего теоретического анализа. Оценка ширины линии SASE, показанная оранжевой пунктирной линией 3 (в online версии), оказывается заметно меньше экспериментального и рассчитанного значений.

\section{4. Возможность каскадного усиления гармоник в SwissFEL}

SwissFEL - последний из введенных в строй рентгеновских ЛСЭ; он был разработан и построен с минимальными затратами в короткие сроки [25-29]. В нем используются электроны с самой низкой для рентгеновских ЛСЭ энергией: $E=5.8 \mathrm{GeV}$; разброс энергии тоже рекордно мал: $\sigma_{e}^{a b s}=350 \mathrm{keV}$, мал также эмиттанс: $\varepsilon_{x, y}=0.4 \mathrm{mmmrad}$ [25], и ток $I_{0}=2.7 \mathrm{kA}$ [29] по сравнению со значениями в других рентгеновских ЛСЭ. В SwissFEL используются ондуляторы с коротким периодом $\lambda_{u}=1.5 \mathrm{~cm}$ и малым номинальным значением ондуляторного параметра $k=1.2$. В последнем эксперименте на SwissFEL [29] излучалась длина волны $\lambda \sim 0.1 \mathrm{~nm}$ при номинальном токе $I=2 \mathrm{kA}$; измеренный эмиттанс, $\varepsilon_{x, y}=0.2 \mathrm{mmmrad}$, оказался даже меньше заданного, а разброс энергий в пучке, $\sigma_{e}=0.0125 \%$ несколько выше ожидаемого. Мощность излучения, доложенная в [29] по результатам этого эксперимента, 


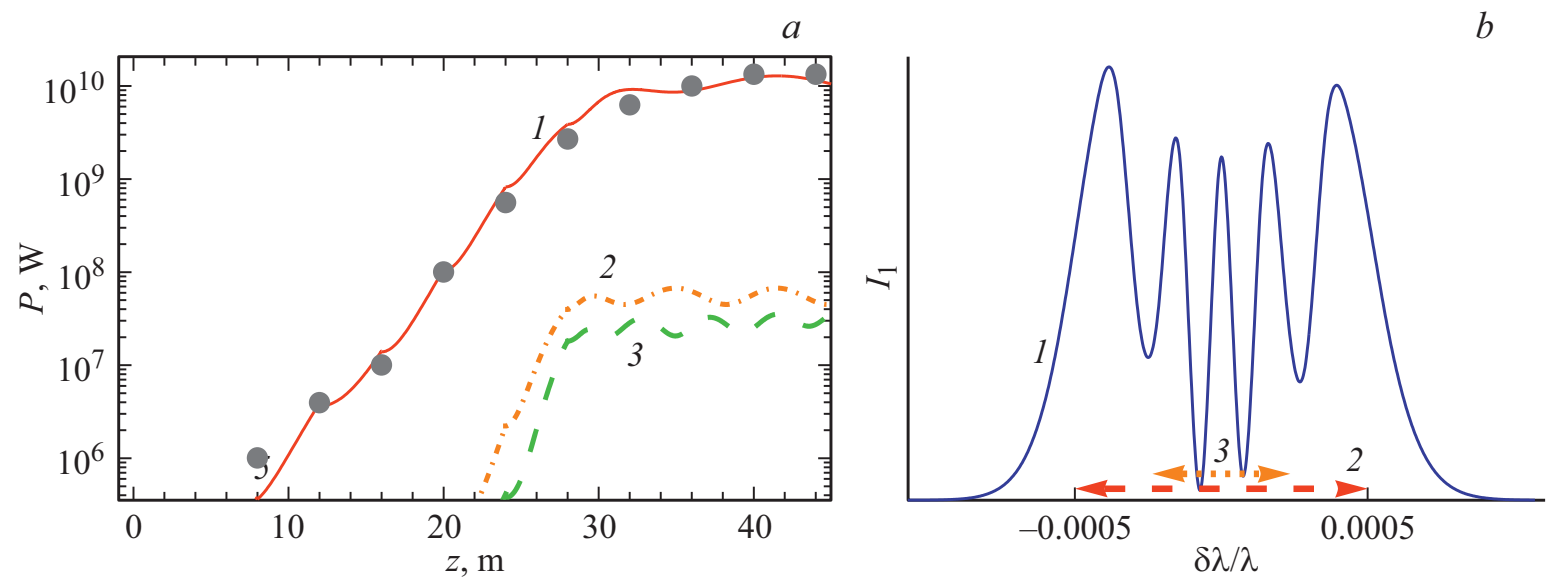

Рис. 2. $a-$ эволюция мощности гармоник в ЛСЭ SwissFEL с $E=5.8 \mathrm{GeV}, I_{0}=2.0 \mathrm{kA}, \sigma_{e}=0.0125 \%$. Гармоники ЛСЭ: $1-\lambda_{1}=0.1 \mathrm{~nm}, 2-\lambda_{2}=0.05 \mathrm{~nm}, 3-\lambda_{3}=0.033 \mathrm{~nm} . b-$ спектральная плотность излучения $\lambda=0.1 \mathrm{~nm}$ ЛCЭ SwissFEL: 1 - теоретическая линия спектра, 2 - средняя измеренная спектральная ширина линии, 3 - оценка ширины линии SASE.

показана точками на рис. 2, $a$; рассчитанная нами аналитически мощность гармоник показана цветными линиями (в online версии). Отметим отличное совпадение экспериментальных и теоретических результатов (ср. сплошную красную линию 1 (в online версии) и точки на рис. 2,a). Также обращает на себя внимание относительно сильное излучение гармоник (зеленая штриховая линия 3 (в online версии) и оранжевая штрихпунктирная линия 2 (в online версии) на рис. $2, a$ ), особенно с учетом малого значения параметра дипольности ондуляторов $k$. Рассчитанная аналитически спектральная плотность излучения, $\Delta \lambda / \lambda \sim 0.1 \%$, находится точно в заявленном для этого ЛСЭ диапазоне $\sim 0.05-0.15 \%$ [26]. Рассчитанная нами ширина линии спектра показана синей линией 1 (в online версии) на рис. 2, $b$, где для сравнения показаны также ширина, измеренная в эксперименте (красная штриховая линия 2 (в online версии)), и оценка для SASE (оранжевая пунктирная линия 3 (в online версии)). Теоретическая ширина отлично согласуется с экспериментом, что подтверждает верность примененного аналитического формализма с использованием обобщенных специальных функций. Оценка для SASE: $\delta \lambda / \lambda \approx \sqrt{\rho \lambda_{u} / L_{s}} \approx 0.0005$, согласуется со значением параметра Пирса $\rho_{1} \approx 0.0005$ и оказывается несколько меньше измеренной спектральной плотности (см. рис. $2, b)$.

Мы предлагаем использовать изменяемый параметр дипольности ондуляторов SwissFEL и выделить группирователь электронов с $k=1.8$ и усилитель второй гармоники с $k=0.7874$. Аналитический расчет дает предполагаемую мощность излучения SwissFEL в режиме HLSS на рис. 3.

Отличное качество пучка с низким эмиттансом и разбросом энергий, позволяет удовлетворить требованиям $\varepsilon / \lambda / 4 \pi, \sigma_{e} \leq \rho / 2$ на первых 16 m ондуляторов. Таким образом, банчер может включать четыре ондуляторных секции. На выходе банчера группировка электронов на длине волны второй гармоники дает большую эффективную мощность для усилителя. Длину усилителя можно

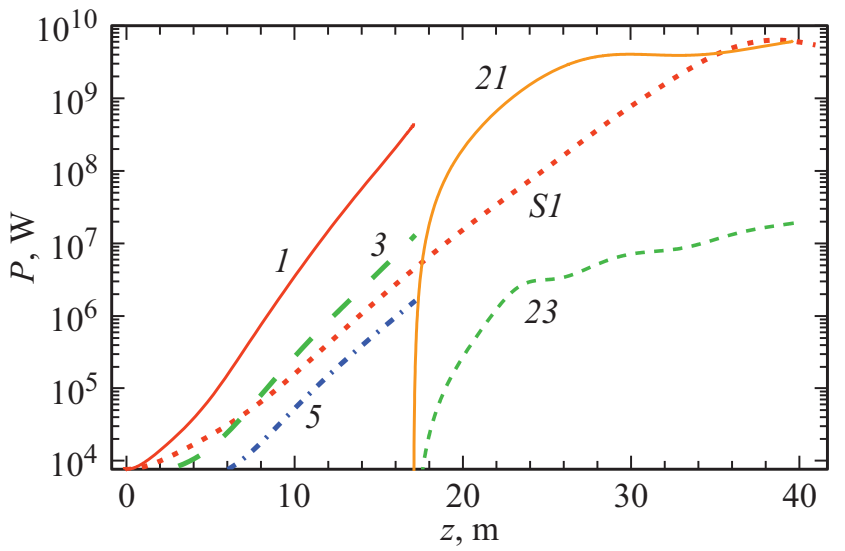

Рис. 3. Эволюция мощности в ЛСЭ SwissFEL в режимax SASE и HLSS с усилением второй гармоники банчера, $E=5.8 \mathrm{GeV}, I_{0}=2.7 \mathrm{kA}$; гармоники банчера: 1 $\lambda_{1}=0.14 \mathrm{~nm}, 3-\lambda_{3}=0.05 \mathrm{~nm}, 5-\lambda_{5}=0.03 \mathrm{~nm}$; гармоники усилителя: $21-\lambda_{1 \times 2}=0.07 \mathrm{~nm}, 23-\lambda_{n=3 \times 2}=0.023 \mathrm{~nm}$; основной тон SASE: $S 1-\lambda_{\mathrm{SASE}, n=1}=0.07 \mathrm{~nm}$.

ограничить двумя-тремя секциями; насыщение наступает уже на 25 m полной длины всех ондуляторов ЛСЭ (см. рис. 3). Для сравнения насыщение ЛСЭ в режиме SASE на той же длине волны $\lambda=0.07 \mathrm{~nm}$ наступает на $\sim 32 \mathrm{~m}$ (см. красную пунктирную линию $S 1$ (в online версии) на рис. 3), что существенно больше, чем в режиме HLSS усиления второй гармоники ЛСЭ.

\section{5. Возможности использования гармоник в ЛСЭ LCLS-II}

В 2009 г. был введен в строй первый рентгеновский ЛCЭ LCLS [10-12]. Теоретический анализ его излучения проводился во многих работах, например, [42,47] и др. LCLS имеет отличные спектральные характеристики даже на сегодняшний день. Сразу после ввода его в 


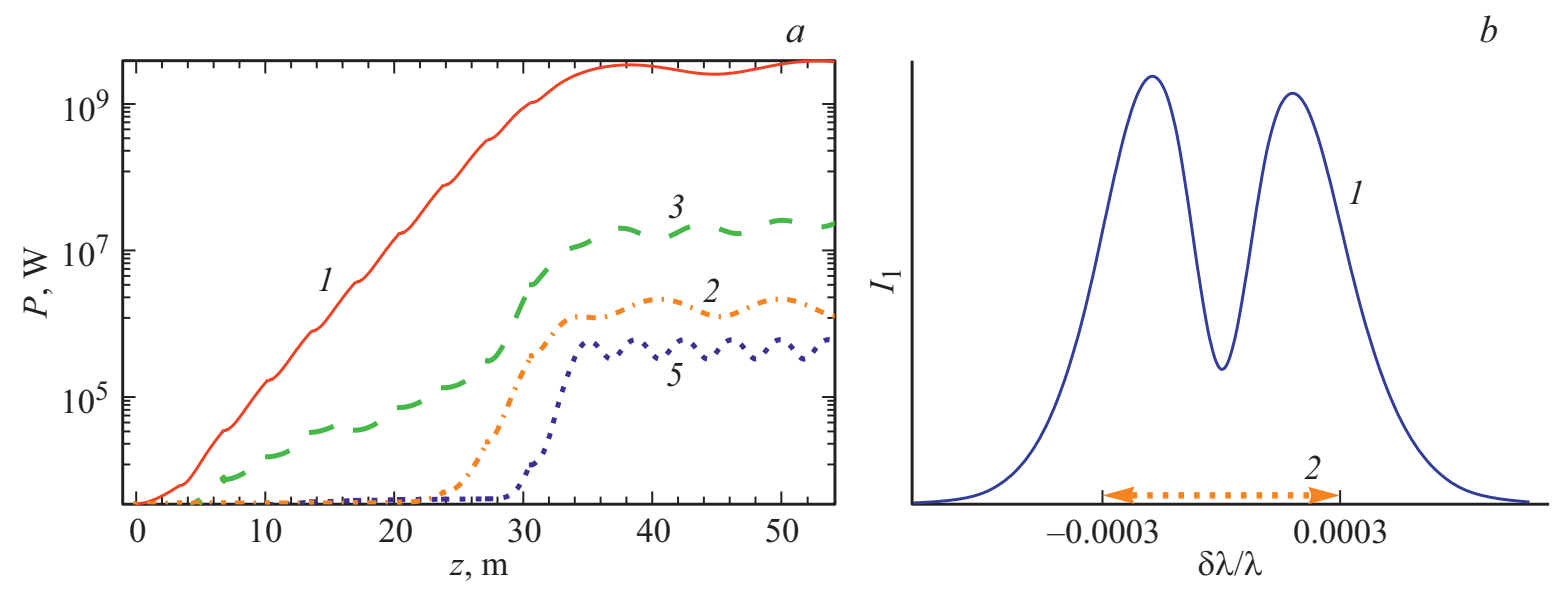

Рис. 4. $a-$ эволюция мощности гармоник в ЛСЭ LCLS-II с $\lambda_{1}=0.378 \mathrm{~nm}$ в режиме SASE: $E=4 \mathrm{GeV}, I_{0}=700 \mathrm{~A}$. Гармоники: $1-\lambda_{n=1}=0.378 \mathrm{~nm}, 2-\lambda_{n=2}=0.189 \mathrm{~nm}, 3-\lambda_{n=3}=0.126 \mathrm{~nm}, 5-\lambda_{n=5}=0.076 \mathrm{~nm} . b-$ спектральная плотность излучения $\lambda=0.378 \mathrm{~nm}$ ЛСЭ LCLS-II: 1 - теоретическая линия спектра, $2-$ оценка ширины линии SASE.

строй начались работы по постройке ЛСЭ LCLS-II с частотой повторения импульсов $1 \mathrm{MHz}$. В нем используется пучок электронов с меньшей энергией $E=4 \mathrm{GeV}$ для генерации рентгеновского излучения с длинами волн $6.2-0.25 \mathrm{~nm}$. Планируется также использование пучка электронов с энергией $E=8 \mathrm{GeV}$ для генерации излучения с длиной волны менее $1 \AA$ в LCLS-II-HE. Большой массив данных по LCLS-II содержится в [30]. Пробные численные симуляции работы LCLS-II проводились в [31-33]. В LCLS-II и II-HE устанавливаются две ондуляторные линии с изменяемым параметром $k$. Для генерации мягкого рентгеновского излучения с $\lambda=6.2-0.95 \mathrm{~nm}$ будут использоваться ондуляторы с периодом $\lambda_{u}=3.9 \mathrm{~cm}$ и полем силой до $\sim 1.5 \mathrm{~T}$; для жесткого рентгеновского излучения с $\lambda=1.2-0.25 \mathrm{~nm}$, будут использоваться ондуляторы с периодом $\lambda_{u}=2.6 \mathrm{~cm}$ и полем до $\sim 1.1 \mathrm{~T}$; минимальный зазор в них $\sim 7 \mathrm{~mm}$. Пучок электронов для LCLS-II имеет малые эмиттанс и разброс энергий. Они зависят от тока и заряда банча: $\varepsilon_{x, y} \approx 0.17 \mu \mathrm{m}, \sigma_{e} \approx 0.4 \mathrm{MeV}$ с током $I \sim 400 \mathrm{~A}$ и зарядом $Q=20 \mathrm{nC}, \varepsilon_{x} \approx 0.37 \mu \mathrm{m}, \varepsilon_{y} \approx 0.45 \mu \mathrm{m}$ и $\sigma_{e} \approx 0.46 \mathrm{MeV}$ с током $I \sim 700$ А и зарядом $Q=100 \mathrm{nC}$, и $\varepsilon_{x, y} \approx 0.45 \mu \mathrm{m}, \sigma_{e} \approx 0.36 \mathrm{MeV}$ с током $I \sim 850 \mathrm{~A} \mathrm{и}$ зарядом $Q=300 \mathrm{nC}[30-33]$. Мы провели моделирование возможного излучения гармоник на LCLS-II в различных режимах: SASE, HLSS и с подавленным основным тоном между ондуляторами. Ниже мы представили результаты для излучения LCLS-II на длинах волн $\lambda=0.38 \mathrm{~nm}$ на рис. $4, a$ и $\lambda=0.25 \mathrm{~nm}$ на рис. $5, a$ с номинальными током $I=700 \mathrm{~A}$, энергией электронов $E=4 \mathrm{GeV}$, и разбросом энергии $\sigma_{e} \approx 0.46 \mathrm{MeV}$, эмиттансом $\varepsilon_{x, y} \approx 0.45 \mu \mathrm{m}$. На рис. 4, $a$ представлена смоделированная эволюция излучения основного тона с $\lambda_{1}=0.378 \mathrm{~nm}$ и его гармоник; отличное качество пучка и ондуляторов позволяет надеяться на гармоники до третьей и, возможно, даже пятой. На рис. 4, $b$ представлена теоретическая ширина линии спектра 1 и оценка ширины линии SASE 2. Ширина спектральной линии на рис. $4, b \Delta \lambda \approx 0.25 \mathrm{pm}$, спектральная плотность $\Delta \lambda / \lambda \approx 0.0007$.

Мы проанализировали возможность использования изменяемого параметра дипольности для реализации раздельного банчера и усилителя гармоник в ЛСЭ LCLS-II по аналогии с тем, как это сделано в ЛСЭ FLASH 2 (см. разд. 4). В частности, на рис. 5, $а$ представлена эволюция мощности излучения с минимально возможной на LCLS-II длиной волны $\lambda=0.248 \mathrm{~nm}$ в режимах ЛСЭ SASE и HLSS с усилением третьей гармоники банчера. Ширина спектральной линии, представленная на рис. $5, b: \Delta \lambda \approx 0.08 \mathrm{pm}$, ее спектральная плотность $\Delta \lambda / \lambda \approx 0.0003$, оценка для SASE хорошо соответствует этому значению $\delta \lambda / \lambda \approx 0.0004$.

Исследование показало отличную группировку электронов в ондуляторах банчера (до $20 \mathrm{~m}$ ) на длине волны его третьей гармоники $\lambda_{3}=0.248 \mathrm{~nm}$, что дает быстрый рост мощности гармоники (см. зеленую штриховую линию 3 (в online версии) на рис. 5, $a$ до $20 \mathrm{~m}$ ). Однако ее усиление в следующих ондуляторных секциях, настроенных в резонанс, происходит медленно (см. оранжевую сплошную линию 31 (в online версии) на рис. 5, a); насыщение начинается примерно на $20 \mathrm{~m}$ раньше чем в SASE, но полная мощность достигается на сравнимой длине, после $60 \mathrm{~m}$. Таким образом, использование изменяемого параметра $k$ в ондуляторах: в банчере $k=2.24$ и в усилителе третьей гармоники $k=0.6$, в этом ЛСЭ дает более ранний рост мощности (рис. 5, $a$ до $45 \mathrm{~m}$ ). Однако медленный рост мощности в усилителе, особенно в конце ЛСЭ после $45 \mathrm{~m}$ (ср. оранжевую сплошную 31 и красную пунктирную $S 1$ линии (в online версии) после $45 \mathrm{~m}$ ) не дает полностью реализовать достоинства выделенного банчера в LCLS-II.

В этом контексте представляется интересным рассмотреть альтернативную настройку ЛСЭ LCLS-II c нарушением группировки электронов на длинах волн основного тона $\lambda_{1}=0.744 \mathrm{~nm}$ между ондуляторами с $k=2.24$. Идея нарушения банчинга основного тона 


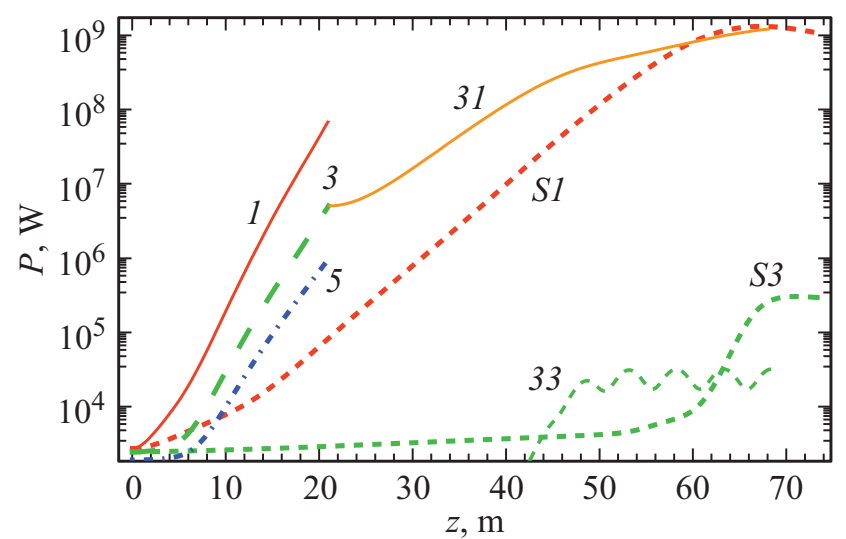

$b$

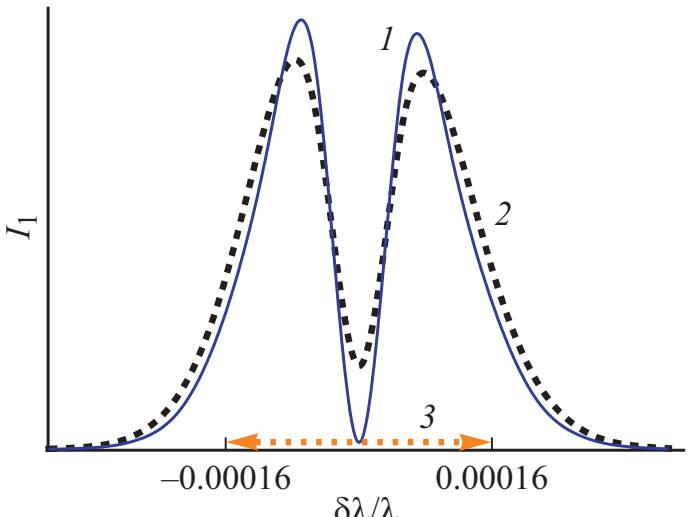

Рис. 5. $a-$ эволюция мощности гармоник в ЛСЭ LCLS-II в режимах SASE и HLSS с усилением второй гармоники банчера $\lambda=0.25 \mathrm{~nm}, E=4 \mathrm{GeV}, \sigma_{e}=0.46 \mathrm{MeV}, I_{0}=700$ A. Гармоники банчера: $1-\lambda_{1}=0.744 \mathrm{~nm}, 3-\lambda_{3}=0.25 \mathrm{~nm}, 5-$ $\lambda_{n=5}=0.15 \mathrm{~nm}$; гармоники усилителя: $31-\lambda_{n=1 \times 3}=0.25 \mathrm{~nm}, 33-\lambda_{n=3 \times 3}=0.08 \mathrm{~nm}$; гармоники SASE: $S 1-\lambda_{\mathrm{SASE}, n=1}=0.25 \mathrm{~nm}$, $S 3-\lambda_{\mathrm{SASE}, n=3}=0.08 \mathrm{~nm}$. $b-$ спектральная плотность излучения $\lambda=0.25 \mathrm{~nm}$ ЛСЭ LCLS-II: $1-$ усилитель третьей гармоники, 2 - SASE, 3 - оценка ширины линии SASE.
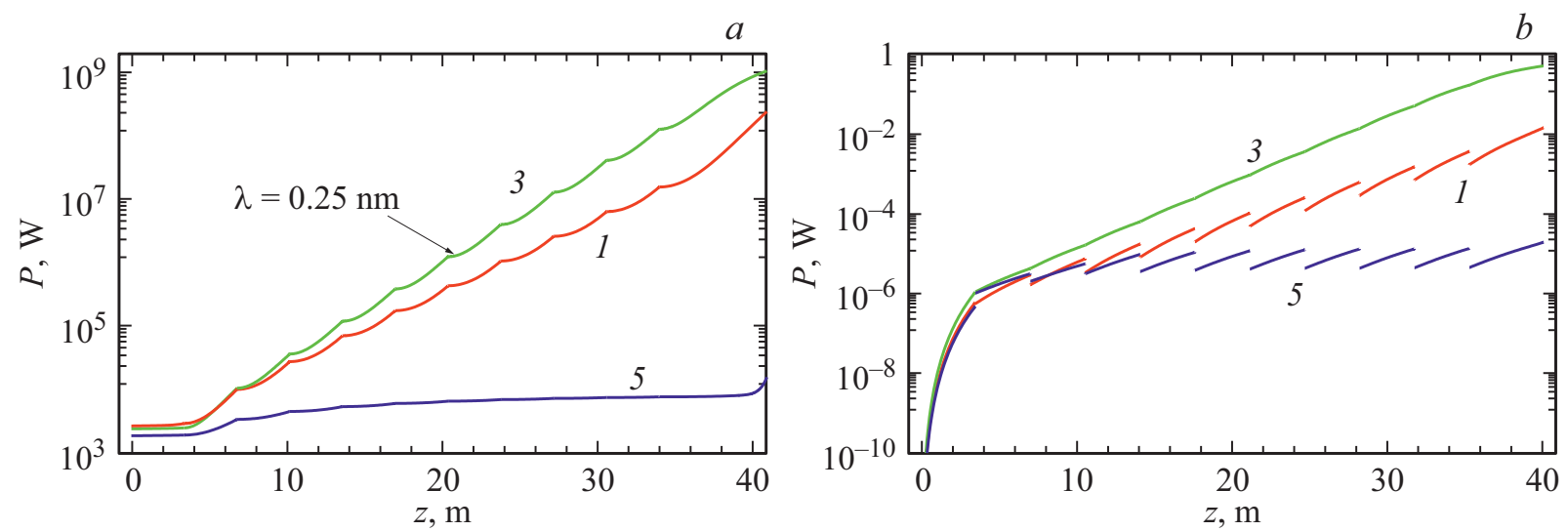

Рис. 6. Эволюция мощности и банчинга гармоник в ЛСЭ LCLS-II с подавленным основным тоном с $\lambda_{1}=0.744 \mathrm{~nm}$, путем нарушения банчинга между секциями ондуляторов; излучается $\lambda_{3}=0.25 \mathrm{~nm} ; a-$ мощность, $b-$ банчинг. Гармоники ЛСЭ: $1-n=1,3-n=3,5-n=5$.

впервые была предложена в [70,71]: между ондуляторами осуществляется фазовый сдвиг фотонов относительно электронов на $k \pi / n$, где $k=2,4,6$. Это нарушает группировку на длине волны основного тона и не затрагивает банчинг третьей гармоники во всех ондуляторах по всей длине ЛСЭ. Используя феноменологическое описание ЛСЭ с расфазировкой, ранее успешно примененное к описанию других ЛСЭ в [72-74], мы рассчитали эволюцию мощности и банчинга в ондуляторах LCLS-II. Рост мощности показан на рис. 6, $a$; эволюция банчинга на рис. $6, b$.

Сравнение роста мощности излучения с $\lambda=0.25 \mathrm{~nm}$ в ондуляторах на рис. 5, $a$ с ростом мощности третьей гармоники на той же длине волны в ондуляторах с подавленным основным тоном на рис. 6, $a$, демонстрирует очевидные преимущества последнего решения. Разрывы банчинга первой гармоники (красная линия 1 (в online версии) на рис. $6, b)$ делают его рост и рост соответству- ющей мощности излучения (красная линия 1 (в online версии) на рис. $6, a)$ медленнее, чем рост третьей гармоники (ср. с зеленой линией 3 (в online версии) на рис. 6). В результате третья гармоника оказывается сильнее первой (см. рис. $6, a)$, а ее усиление в ондуляторах с $k=2.24$ превосходит усиление на той же длине волны в ондуляторах с $k=0.6$. Сравним эволюцию мощности излучения на длине волны $\lambda=0.25 \mathrm{~nm}$ в ЛСЭ в режимах SASE, HLSS (рис. 5, $a$ ) и с подавленным основным тоном (рис. 6,a). Сравнение оранжевой сплошной линии 31 и красной пунктирной линии $S 1$ (в online версии) на рис. 5, $a$ и зеленой линии 3 (в online версии) на pис. $6, a$ показывает, что длина насыщения для излучения на длине волны $\lambda=0.25 \mathrm{~nm}$ составляет: $L_{s} \sim 65 \mathrm{~m}$ для основного тона SASE, $L_{s} \sim 50$ m для HLSS с каскадным усилением третьей гармоники (рис. 5,a), и $L_{s} \sim 40 \mathrm{~m}$ для третьей гармоники SASE с подавленным основным тоном (рис. $6, a)$. 


\section{6. Результаты и выводы}

Проведено теоретическое исследование излучения гармоник рентгеновских ЛСЭ FLASH2, SwissFEL, и строящегося ЛСЭ LCLS-II. Для ЛСЭ FLASH2 и SwissFEL проведено сравнение полученных спектральных характеристик с экспериментальными данными и подтверждено соответствие теории и экспериментов. Проведено сравнение полученных теоретических результатов эволюции мощности в режимах самоусиления спонтанного излучения SASE и усиления третьей гармоники банчера с имеющимися данными для ЛСЭ FLASH2. Рассмотрена возможность использования выделенного банчера и усилителя его гармоник в ЛСЭ SwissFEL, a также в строящемся ЛСЭ LCLS-II. Для LCLS-II исследована также возможность излучения третьей гармоники в режиме подавления излучения основного тона путем нарушения банчинга между ондуляторами.

В ЛСЭ FEL FLASH2 с изменяемым параметром дипольности рассмотрена генерация на длине волны $\lambda=11 \mathrm{~nm}$ в режимах SASE (self-amplified spontaneous emission) и HLSS (harmonic lasing self-seed) с затравкой третьей гармоникой банчера. Показано, что мощность 3-й гармоники за счет более эффективной группировки электронов на ее длине волны в банчере растет быстрее, чем основной тон SASE на той же длине волны. На 25 m ондуляторов мощность ЛСЭ HLSS в пять раз больше, а насыщение наступает на десять метров раньше, чем в ЛСЭ SASE. Причина этого в значении параметра дипольности банчера, позволяющего осуществить эффективную группировку электронов на длине волны гармоники.

В ЛСЭ SwissFEL используется пучок электронов относительно низкой энергии $E=5.8 \mathrm{GeV}$, с малым разбросом и эмиттансом. Это позволяет получить большую мощность излучения на длине волны $\lambda \sim 0.1 \mathrm{~nm}$ и ее гармоник в ондуляторах с относительно малым значением $k$. ЛСЭ имеет короткую длину насыщения: $L_{s} \sim 35 \mathrm{~m}$. На основе теоретического расчета предложено с имеющимися ондуляторами с изменяемым параметром дипольности выделить банчер с $k=1.8$ и усилитель с $k=0.787$ для второй гармоники на длине волны $\lambda_{2}=0.07 \mathrm{~nm}$. При этом длина насыщения при максимальном токе $I_{0}=2.7$ А уменьшается до $\sim 28 \mathrm{~m}$, а длина волны будет меньше стандартной на $30 \%$.

Изучена возможность генерации гармоник в строящемся ЛСЭ LCLS-II с пучком электронов низкой энергии $E=4 \mathrm{GeV}$. Расчет показал, что малые эмиттанс и разброс энергий должны обеспечить отличные спектральные характеристики излучения, в том числе для гармоник. Сравнительный анализ режимов SASE и HLSS с усилением третьей гармоники банчера в ондуляторах с изменяемым параметром $k$ показал значительно более ранний рост мощности в ЛСЭ HLSS с банчером с $k=2.24$, чем в SASE на той же длине волны. Однако из-за малого значения $k=0.6$ в ондуляторах усилителя рост мощности в них происходит медленно и длина насыщения ЛСЭ HLSS сравнима с длиной SASE. Это не позволяет в полной мере реализовать в LCLS-II преимущества группировки электронов на длине волны третьей гармоники банчера. Поэтому предложено осуществить усиление третьей гармоники в ондуляторах с $k=2.24$ по всей длине ЛСЭ и использовать нарушение банчинга основного тона между ондуляторами для его подавления. Показано, что в этом случае происходит эффективное уменьшение мощности основного тона ниже мощности третьей гармоники ЛСЭ. При этом излучение третьей гармоники на длине волны $\lambda_{3}=0.25 \mathrm{~nm}$ в LCLS-II достигает мощности $>1 \mathrm{GW}$ и ее насыщение наступает на длине $L_{s} \sim 40 \mathrm{~m}$, что существенно короче по сравнению с $L_{s} \sim 65 \mathrm{~m}$ для ЛСЭ SASE и $L_{s} \sim 50 \mathrm{~m}$ для ЛСЭ с каскадным усилением гармоник.

Исследование показывает, что в ЛСЭ последнего поколения улучшенное качество пучков позволяет генерировать рентгеновские излучения гармоник в ондулятоpax c коротким периодом и малым параметром дипольности, используя электроны более низкой энергии.

\section{Благодарности}

Авторы благодарят проф. А.В. Борисова, проф. В.Г. Багрова и гл.н.с. А.Е. Лобанова за полезные советы и обсуждение.

\section{Конфликт интересов}

Автор заявляет, что у него нет конфликта интересов.

\section{Список литературы}

[1] J.M. Madey. J. Appl. Phys., 42, 1906 (1971).

[2] D.A.G. Deacon, L.R. Elias, J.M.J. Madey, G.J. Ramian, H.A. Schwettman, T.I. Smith. Phys. Rev. Lett., 38, 892 (1977).

[3] V.L. Ginzburg. Isv. AN SSSR Fiz., 11, 1651 (1947).

[4] H. Motz., W. Thon, R.N.J. Whitehurst. Appl. Phys., 24, 826 (1953).

[5] H. Motz. J. Appl. Phys., 22, 527 (1951). https://doi.org/10.1038/nphoton.2010.239

[6] B.W.J. Mc Neil, N.R. Thompson. Nature Photonics, 4, 814 (2010). https://doi.org/10.1038/nphoton.2010.239

[7] C. Pellegrini, A. Marinelli, S. Reiche. Rev. Mod. Phys., 88, 015006 (2016). https://doi.org/10.1103/RevModPhys.88.015006

[8] K.J. Kim, Z. Huang, R. Lindberg, in Principles of Coherent $X$-Ray Radiation (Cambridge University Press, Cambridge CB2 8BS, United Kingdom), DOI: 10.1017/9781316677377

[9] Z. Huang, K.J. Kim. Phys. Rev. ST-AB, 10, 034801 (2007). https://doi.org/10.1103/PhysRevSTAB.10.034801

[10] P. Emma, R. Akre, J. Arthur et al. Nature Photonics, 4, 641 (2010). https://doi.org/10.1038/nphoton.2010.176

[11] P. Emma, First lasing of the LCLS X-ray FEL at $1.5 \AA$, TH3PBI01, Proceedings of PAC09, Vancouver, BC, Canada, (2009).

[12] D. Ratner, A. Brachmann, F.J. Decker, Y. Ding, D. Dowell, P. Emma, A. Fisher, J. Frisch, S. Gilevich, Z. Huang, P. Hering, R. Iverson, J. Krzywinski, H. Loos, M. Messerschmidt, H.D. Nuhn, T. Smith, J. Turner, J. Welch, 
W. White, J. Wu. Phys. Rev. ST-AB 14, 060701 (2011). https://doi.org/10.1103/PhysRevSTAB.14.060701

[13] L. Yu, M. Babzien, I. Ben-Zvi, L.F. Di Mauro, A. Doyuran, W. Graves, E. Johnson, S. Krinsky, R. Malone, I. Pogorelsky, J. Skaritka, G. Rakowsky, L Solomon, X.J. Wang, M. Woodle, V. Yakimenko, S.G. Biedron, J.N. Galayda, E. Gluskin, J. Jagger, V. Sajaev, I. Vasserman. Science, 289, 932 (2000).

[14] T. Shaftan, L.-H. Yu, Phys. Rev. E, 71, 046501 (2005). https://doi.org/10.1103/PhysRevE.71.046501

[15] D. Xiang, G. Stupakov. Phys. Rev. ST-AB, 12, 030702 (2009). https://doi.org/10.1103/PhysRevSTAB.12.030702

[16] D. Xiang, E. Colby, M. Dunning, S. Gilevich, C. Hast, K. Jobe, D. Mc Cormick, J. Nelson, T.O. Raubenheimer, K. Soong, G. Stupakov, Z. Szalata, D. Walz, S. Weathersby, M. Woodley, P.-L. Pernet. Phys. Rev. Lett., 105, 114801 (2010). https://doi.org/10.1103/PhysRevLett.105.114801

[17] P.R. Ribič, A. Abrami, E.M. Allariaэ. Nature Photonics, 13, 555 (2019). https://doi.org/10.1038/s41566-019-0427-1

[18] G. Dattoli, P.L. Ottaviani, S. Pagnutti. J. Appl. Phys., 97, 113102 (2005). https://doi.org/10.1063/1.1886890

[19] R. Bonifacio, L. De Salvo, P. Pierini. Nucl. Instrum. A, 293, 627 (1990). https://doi.org/10.1016/0168-9002(90)90334-3

[20] K. Zhukovsky. Opt. Comm. 418, 57 (2018). https://doi.org/10.1016/j.optcom.2018.02.039

[21] K. Zhukovsky. J. Appl. Phys., 122, 233103 (2017). https://doi.org/10.1063/1.5001794

[22] K. Zhukovsky. EPL, 119, 34002 (2017). https://doi.org/10.1209/0295-5075/119/34002

[23] O. Hensler, K. Honkavaara, R. Kammering, M. Kuhlmann, E. Ploenjes, J. Roensch-Schulenburg, E. Schneidmiller, S. Schreiber, K. Tiedtke, M. Tischer, R. Treusch, M. Vogt, W. Wurth, M. Yurkov, J. Zemella. Appl. Sci., 7 (11), 1114 (2017). https://doi.org/10.3390/app7111114

[24] E.A. Schneidmiller, B. Faatz, M. Kuhlmann, J. Roensch-Schulenburg, S. Schreiber, M. Tischer, M.V. Yurkov. Phys. Rev. ST-AB, 20, 020705 (2017).

[25] C.J. Milne, T. Schietinger, M. Aiba, A. Alarcon, J. Alex, A. Anghel, V. Arsov, C. Beard, P. Beaud, S. Bettoni, M. Bopp, H. Brands, M. Brönnimann, I. Brunnenkant, M. Calvi, A. Citterio, P. Craievich, M.C. Divall, M. Dällenbach, M. D'Amico, A. Dax, Y. Deng, A. Dietrich, R. Dinapoli, E. Divall, S. Dordevic, S. Ebner, C. Erny, H. Fitze, U. Flechsig, R. Follath, F. Frei, F. Gärtner, R. Ganter, T. Garvey, Z. Geng, I. Gorgisyan, C. Gough, A. Hauff, C.P. Hauri, N. Hiller, T. Humar, S. Hunziker, G. Ingold, R. Ischebeck, M. Janousch, P. Jurani'c, M. Jurcevic, M. Kaiser, B. Kalantari, R. Kalt, B. Keil, C. Kittel, G. Knopp, W. Koprek, H.T. Lemke, T. Lippuner, D.L. Sancho, F. Löhl, C. Lopez-Cuenca, F. Märki, F. Marcellini, G. Marinkovic, I. Martiel, R. Menzel, A. Mozzanica, K. Nass, G.L. Orlandi, C.O. Loch, E. Panepucci, M. Paraliev, B. Patterson, B. Pedrini, M. Pedrozzi, P. Pollet, C. Pradervand, E. Prat, P. Radi, J.-Y. Raguin, S. Redford, J. Rehanek, J. R'ehault, S. Reiche, M. Ringele, J. Rittmann, L. Rivkin, A. Romann, M. Ruat, C. Ruder, L. Sala, L. Schebacher, T. Schilcher, V. Schlott, T. Schmidt, B. Schmitt, X. Shi, M. Stadler, L. Stingelin, W. Sturzenegger, J. Szlachetko, D. Thattil, D.M. Treyer, A. Trisorio, W. Tron, S. Vetter, C. Vicario, D. Voulot, M. Wang, T. Zamofing, C. Zellweger, R. Zennaro, E. Zimoch, R. Abela, L. Patthey, H.-H. Braun. Appl. Sci. 7, 720 (2017). https://doi.org/10.3390/app7070720
[26] R. Abela, P. Beaud, J.A. van Bokhoven, M. Chergui, T. Feurer, J. Haase, G. Ingold, S.L. Johnson, G. Knopp, H. Lemke, C.J. Milne, B. Pedrini, P. Radi, G. Schertler, J. Standfuss, U. Staub, L. Patthey. Struct. Dyn., 4, 061602 (2017). https://doi.org/10.1063/1.4997222

[27] P. Juranić, J. Rehanek, C.A. Arrell, C. Pradervand, R. Ischebeck, C. Erny, P. Heimgartner, I. Gorgisyan, V. Thominet, K. Tiedtke, A. Sorokin, R. Follath, M. Makita, G. Seniutinas, C. David, C.J. Milne, H. Lemke, M. Radovic, C.P. Hauri, L. Patthey. J. Synchrotron Rad., 26, 906 (2019). https://doi.org/10.1107/S1600577519005654

[28] R. Abela, A. Alarcon, J. Alex, C. Arrell, V. Arsov, S. Bettoni, M. Bopp, C. Bostedt, H.-H. Braun, M. Calvi, T. Celcer, P. Craievich, A. Dax, P. Dijkstal, S. Dordevic, E. Ferrari, U. Flechsig, R. Follath, F. Frei, N. Gaiffi, Z. Geng, C. Gough, N. Hiller, S. Hunziker, M. Huppert, R. Ischebeck, H. Jöhri, P. Juranic, R. Kalt, M. Kaiser, B. Keil, C. Kittel, R. Künzi, T. Lippuner, F. Löhl, F. Marcellini, G. Marinkovic, C. Ozkan Loch, G.L. Orlandi, B. Patterson, C. Pradervand, M. Paraliev, M. Pedrozzi, E. Prat, P. Ranitovic, S. Reiche, C. Rosenberg, S. Sanfilippo, T. Schietinger, T. Schmidt, K. Schnorr, C. Svetina, A. Trisorio, C. Vicario, D. Voulot, U. Wagner, H.J. Wörner, A. Zandonella, L. Patthey, R. Ganter. J. Synchrotron Rad., 26, 1073 (2019). https://doi.org/10.1107/S1600577519003928

[29] E. Prat, R. Abela, M. Aiba, A. Alarcon, J. Alex, Y. Arbelo, C. Arrell, V. Arsov, C. Bacellar, C. Beard, P. Beaud, S. Bettoni, R. Biffiger, M. Bopp, H.-Heinrich Braun, M. Calvi, A. Cassar, T. Celcer, M. Chergui, P. Chevtsov, C. Cirelli, A. Citterio, P. Craievich, M.C. Divall, A. Dax, M. Dehler, Y. Deng, A. Dietrich, P. Dijkstal, R. Dinapoli, S. Dordevic, S. Ebner, D. Engeler, C. Erny, V. Esposito, E. Ferrari, U. Flechsig, R. Follath, F. Frei, R. Ganter, T. Garvey, Z. Geng, A. Gobbo, C. Gough, A. Hauff, C. P. Hauri, N. Hiller, S. Hunziker, M. Huppert, G. Ingold, R. Ischebeck, M. Janousch, P.J.M. Johnson, S.L. Johnson, P. Juranić, M. Jurcevic, M. Kaiser, R. Kalt, B. Keil, D. Kiselev, C. Kittel, G. Knopp, W. Koprek, M. Laznovsky, H.T. Lemke, D.L. Sancho, F. Löhl, A. Malyzhenkov, G.F. Mancini, R. Mankowsky, F. Marcellini, G. Marinkovic, I. Martiel, F. Märki, C.J. Milne, A. Mozzanica, K. Nass, G. Luca Orlandi, C. Ozkan Loch, M. Paraliev, B. Patterson, L. Patthey, B. Pedrini, M. Pedrozzi, C. Pradervand, P. Radi, J.-Y. Raguin, S. Redford, J. Rehanek, S. Reiche, L. Rivkin, A. Romann, L. Sala, M. Sander, T. Schietinger, T. Schilcher, V. Schlott, T. Schmidt, M. Seidel, M. Stadler, L. Stingelin, C. Svetina, D.M. Treyer, A. Trisorio, C. Vicario, D. Voulot, A. Wrulich, S. Zerdane, E. Zimoch. Nature Photon., 14, 748 (2020). https://doi.org/10.1038/s41566-020-00712-8

[30] Электронный pecypc. Режим доступа: https://portal.slac. stanford.edu/sites/lcls_public/lcls_ii/acc_phy/Lists/technotes/ Public_view.aspx

[31] T.O. Raubenheimer, in 60th ICFA Advanced Beam Dynamics Workshop on Future Light Sources FLS 2018 (Shanghai, China, 2018), doi: 10.18429/JACoW-FLS2018-MOP1WA02

[32] G. Marcus, J. Qiang, LCLS-II SCRF Start-to-end Simulations and Global Optimization as of September 2016, SLAC National Accelerator Lab, LCLS-II TN-17-04, February 2017.

[33] G. Marcus, J. Qiang, LCLS-II SCRF start-to-end simulations as of August 2015, SLAC National Accelerator Lab, LCLS-II TN-15-33, February 2017. 
[34] В.Г. Багров, Г.С. Бисноватый-Коган, В.А. Бордовицын, А.В. Борисов, О.Ф. Дорофеев, Я.В. Япп, Ю.Л. Пивоваров, О.В. Шорохов, В.Ч. Жуковский. Теория излучения релятивистских частиц (Физматлит, М., 2002), 575 c. [V.G. Bagrov, G.S. Bisnovaty-Kogan, V.A. Bordovitsyn, A.V. Borisov, O.F. Dorofeev, Ya.V. Epp, Y.L. Pivovarov, O.V. Shorokhov, V.C. Zhukovsky. 1999 Synchrotron Radiation Theory and Its Development ed V.A. Bordovitsyn (Singapore: Word Scientific), p. 447.

[35] I.M. Ternov, V.V. Mikhailin, V.R. Khalilov, Synchrotron Radiation and its Applications (CRC Press, 1985)

[36] V.G. Bagrov, V.F. Zal'mezh, M.M. Nikitin, V.Y. Epp. Nucl. Instr. Meth. A, 261, 54 (1987).

[37] Н.А. Винокуров, Е.Б. Левичев. УФН, 185, 917 (2015). DOI: $10.3367 /$ UFNr.0185.201509b.0917 [N.A. Vinokurov, E.B. Levichev. Phys. Usp., $58(9), \quad 917 \quad$ (2015) DOI: $10.3367 /$ UFNe.0185.201509b.0917]

[38] Д.Ф. Алферов, Ю.А. Башмаков, Е.Г. Бессонов. ЖТФ, 43 (10), 2126 (1973). [D.F. Alferov, Yu.A. Bashmakov, E.G. Bessonov. Sov. Phys.-Tech. Phys., 18, 1336 (1974).]

[39] D.F. Alferov, Y.A. Bashmakov, E.G. Bessonov, P.A. Cherenkov, K.A. Belovintsev. The Ondulator as a Source of Electromagnetic Radiation. Part. Accel., 9, 223 (1979).

[40] Д.Ф. Алферов, Ю.А. Башмаков, П.А. Черенков. УФН, 157 (3), 389 (1989). [D.F. Alferov, Yu.A. Bashmakov, P.A. Cherenkov. Sov. Phys. Uspekhi, 32 (3), 200 (1989).]

[41] K. Zhukovsky. Res. Phys., 13, 102248 (2019). https://doi.org/10.1016/j.rinp.2019.102248

[42] K.V. Zhukovsky. J. Synchrotron Rad., 26, 1481 (2019). https://doi.org/10.1107/S1600577519008415

[43] К.В. Жуковский. Изв. вузов. Физика, 62 (6), 109 (2019). [K.V. Zhukovsky. Russ. Phys. J., 62 (6), 1043 (2019). https://doi.org/10.1007/s11182-019-01812-x

[44] G. Mishra, A. Sharma. Nucl. Instrum. Meth. Phys. Res. A, 976, 164287 (2020). DOI: 10.1016/j.nima.2020.164287

[45] G. Mishra, A. Sharma, S.M. Khan. Prog. Electromagn. Res. C, 105, 217 (2020). doi: 10.2528/PIERC20052201

[46] К.В. Жуковский., А.М. Калитенко. Изв. вузов. Физика, $62(2), 153$ (2019). [K.V. Zhukovsky, A.M. Kalitenko. Russ. Phys. J., $62(2), 354$ (2019). https://doi.org/10.1007/s11182-019-01719-7

[47] K. Zhukovsky. Opt. Laser Technol., 131, 106311 (2020) . https://doi.org/10.1016/j.optlastec.2020.106311

[48] K. Zhukovsky, I. Fedorov. Symmetry, 13 (1), 135 (2021). https://doi.org/10.3390/sym13010135

[49] G. Dattoli, V.V. Mikhailin, K. Zhukovsky. J. Appl. Phys., 104, 124507 (2008). https://doi.org/10.1063/1.3039094

[50] Д. Даттоли, К.В. Жуковский, В.В. Михайлин. Вестник Московского ун-та. Серия 3: Физика, астрономия, 5, 33 (2009). [G. Dattoli, V.V. Mikhailin, K.V. Zhukovsky. Mosc. Univ. Phys. Bull., 64 (5), 507 (2009). https://doi.org/10.3103/S0027134909050087

[51] К.В. Жуковский. Вестн. Моск. ун-та. Физ. Астрон. 4, 3 (2020). [K.V. Zhukovsky. Mosc. Univ. Phys. Bull., 4, 285 (2020) . https://doi.org/10.3103/S002713492004013X

[52] K. Zhukovsky. Res. Phys., 19, 103361 (2020). https://doi.org/10.1016/j.rinp.2020.103361

[53] K. Zhukovsky. Symmetry, 12, 1258 (2020). https://doi.org/10.3390/sym12081258

[54] K. Zhukovsky. J. Synch. Rad., 27, 1648 (2020). https://doi.org/10.1107/S1600577520012230
[55] К.В. Жуковский. УФН, 191 (3), 318 (2021). DOI: $10.3367 / \mathrm{UFNr}$ 2020.06.038803 [K.V. Zhukovsky. Phys. Uspekhi, 64 (3), (2021). DOI: 10.3367/UFNe.2020.06.038803

[56] P. Schmüser, M. Dohlus, J. Rossbach, C. Behrens. Free-Electron Lasers in the Ultraviolet and X-Ray Regime. In: Springer Tracts in Modern Physics, 258, Cham (ZG), (Springer International Publishing, 2014), DOI: 10.1007/978-3-319-04081-3

[57] R. Bonifacio, C. Pellegrini, L.M. Narducci. Opt. Comm., 50 (6), 373 (1984).

[58] H. Haus. IEEE J. Quant. Electron., 17 (8), 1427 (1981).

[59] S.V. Milton, E. Gluskin, N.D. Arnold, C. Benson, W. Berg, S.G. Biedron, M. Borland, Y.-C. Chae, R.J. Dejus, P.K. Den Hartog, B. Deriy, M. Erdmann, Y.I. Eidelman, M.W. Hahne, Z. Huang, K.-J. Kim, J.W. Lewellen, Y. Li, A.H. Lumpkin, O. Makarov, E.R. Moog, A. Nassiri, V. Sajaev, R. Soliday, B.J. Tieman, E.M. Trakhtenberg, G. Travish, I.B. Vasserman, N.A. Vinokurov, X.J. Wang, G. Wiemerslage, B.X. Yang. Science, 292, 2037 (2001). DOI: $10.1126 /$ science. 1059955

[60] M. Xie. Nucl. Instrum. Methods Phys. Res., Sect. A, 445, 59 (2000). https://doi.org/10.1016/S0168-9002(00)00114-5

[61] M. Xie. in Proceedings of the 1995 Particle AcceleratorConference (IEEE, Piscataway, NJ., 1995), p. 183.

[62] G. Dattoli, L. Giannessi, P.L. Ottaviani, C. Ronsivalle. J. Appl. Phys., 95, 3206 (2004).

https://doi.org/10.1063/1.1645979

[63] G. Dattoli, P.L. Ottaviani, S. Pagnutti, Booklet for FEL Desing: a Collection of Practical Formulae, ENEA Report RT/2007/40/FIM (2007)

[64] G. Dattoli, V.V. Mikhailin, P.L. Ottaviani, K.V. Zhukovsky. J. Appl. Phys., 100 (8), 084507 (2006). https://doi.org/10.1063/1.2357841

[65] G. Dattoli, P.L. Ottaviani. Opt. Commun., 204 (1), 283 (2002). https://doi.org/10.1016/S0030-4018(02)01201-4

[66] L. Giannessi, in Synchrotron Light Sources and Free-Electron Lasers, E.J. Jaeschke et al. (eds.), (Springer International Publishing Switzerland, 2016), DOI: 10.1007/978-3-319-14394-1_3

[67] К.В. Жуковский. Вестн. Моск. ун-та. Физ. Астрон., 5, 60 (2019). [K.V. Zhukovsky. Mosc. Univ. Phys. Bull., 74 (5), 480 (2019). https://doi.org/10.3103/S0027134919050187]

[68] L. Giannessi, D. Alesini, P. Antici, A. Bacci, M. Bellaveglia, R. Boni, M. Boscolo, F. Briquez, M. Castellano, L. Catani, E. Chiadroni, A. Cianchi, F. Ciocci, A. Clozza, M. E. Couprie, L. Cultrera, G. Dattoli, M. Del Franco, A. Dipace, G. Di Pirro, A. Doria, A. Drago, W.M. Fawley, M. Ferrario, L. Ficcadenti, D. Filippetto, F. Frassetto, H. P. Freund, V. Fusco, G. Gallerano, A. Gallo, G. Gatti, A. Ghigo, E. Giovenale, A. Marinelli, M. Labat, B. Marchetti, G. Marcus, C. Marrelli, M. Mattioli, M. Migliorati, M. Moreno, A. Mostacci, G. Orlandi, E. Pace, L. Palumbo, A. Petralia, M. Petrarca, V. Petrillo, L. Poletto, M. Quattromini, J.V. Rau, S. Reiche, C. Ronsivalle, J. Rosenzweig, A.R. Rossi, V. Rossi Albertini, E. Sabia, L. Serafini, M. Serluca, I. Spassovsky, B. Spataro, V. Surrenti, C. Vaccarezza, M. Vescovi, C. Vicario. Phys. Rev. ST-AB, 14, 060712 (2011). https://doi.org/10.1103/PhysRevSTAB.14.060712

[69] K. Zhukovsky, A. Kalitenko. J. Synchrotron Rad., 26, 159 (2019). DOI: $10.1107 /$ S1600577518012444 
[70] B.W.J. Mc Neil, G.R.M. Robb, M.W. Poole, N.R. Thompson. Phys. Rev. Lett., 96, 084801 (2006).

https://doi.org/10.1103/PhysRevLett.96.084801

[71] E.A. Schneidmiller, M.V. Yurkov. Phys. Rev. ST-AB, 15, 080702 (2012)

https://doi.org/10.1103/PhysRevSTAB.15.080702

[72] К.В. Жуковский. Вестн. Моск. ун-та. Физ. Астрон., 5, 18 (2018) [K.V. Zhukovsky. Moscow Univ. Phys. Bull., 74 (3), 308 (2018). https://doi.org/10.3103/S0027134918050193

[73] K. Zhukovsky. J. Optics, 20 (9), 095003 (2018).

DOI: 10.1088/2040-8986/aad6af

[74] К.В. Жуковский, А.М. Калитенко. ЖТФ, 90 (8), 1337 (2020). DOI: 10.21883/JTF.2020.08.49545.280-18 [K.V. Zhukovskii, A.M. Kalitenko. Tech. Phys., 65, 1285 (2020). https://doi.org/10.1134/S106378422008024] 